and Geotechnics

Elsevier Editorial System(tm) for Computers

Manuscript Draft

Manuscript Number: COGE-D-15-00327R1

Title: Vermiculate artefacts in image analysis of granular materials

Article Type: Research Paper

Keywords: granular materials; digital image correlation; vermiculation; sub-pixel

interpolation

Corresponding Author: Dr. Samuel A Stanier,

Corresponding Author's Institution: UWA

First Author: Samuel A Stanier

Order of Authors: Samuel A Stanier; Jelke Dijkstra; Danuta Lesniewska; James P Hambleton; David J White; David Muir Wood

Abstract: Some reported analyses of images of deforming granular materials have generated surprising vermiculate strain features which are difficult to reconcile with the mechanics of deformation of granular matter. Detailed investigation using synthetic images and improved processing of images of laboratory experiments indicates that such features can emerge as a consequence of the image acquisition (sensor, contrast, resolution), the subsequent image correlation implementation, and the user's choice of processing parameters. The two principal factors are: (i) the texture and resolution of the images and (ii) the algorithm used to achieve sub-pixel displacement resolution. Analysis of the images using a sub-pixel interpolation algorithm that is more robust than that used originally eliminates the vermiculate features for images with moderate resolution and texture. However, erroneous features persist in images with low resolution and poor texture. Guidance is provided on ways in which such artefacts can be avoided through improved experimental and image analysis techniques. 


\section{COGE-D-15-00327- Peer Review Comments \& Actions}

The authors would like to thank the reviewers for their helpful comments regarding the paper draft and have made the following amendments. All changes (deletions and additions) made to the original manuscript are clearly marked in the revised paper. We hope the manuscript is now acceptable for publication.

Reviewer 1:

\begin{tabular}{|c|c|}
\hline Comment & Actions \\
\hline $\begin{array}{l}\text { I really enjoyed reading this paper that I } \\
\text { found very interesting. I had only a few } \\
\text { comments for the consideration of the } \\
\text { authors. }\end{array}$ & N/A \\
\hline $\begin{array}{l}\text { I was perhaps a little worried about the } \\
\text { longevity of some of the } \\
\text { recommendations and conclusions. In } \\
\text { an area where I guess that the } \\
\text { technology is changing rapidly, to what } \\
\text { extent are the conclusions robust or are } \\
\text { they a function of the current } \\
\text { technology level? }\end{array}$ & $\begin{array}{l}\text { All recommendations are applicable to any } \\
\text { image-based deformation analysis that } \\
\text { processes subsets of the image pairs, } \\
\text { irrespective of the algorithm employed. } \\
\text { Consequently we believe that the } \\
\text { recommendations and conclusions will } \\
\text { prove to be robust over time and be useful } \\
\text { for the geotechnical research community. }\end{array}$ \\
\hline $\begin{array}{l}\text { In particular the paper sometimes } \\
\text { seemed to recommend not collecting } \\
\text { too much data/information, for example } \\
\text { in the overlap (Rule 5) or the number of } \\
\text { pixels per particle (Rule 2). This was a } \\
\text { little surprising as I can't believe that } \\
\text { more data is worse than less and it } \\
\text { reminds me of similar arguments in the } \\
\text { early days of measuring small strain } \\
\text { stiffness in lab tests, where it was } \\
\text { commonly argued that the plots were } \\
\text { less noisy if fewer data were collected! } \\
\text { Surely this must be a function of the } \\
\text { current state of the art of the images } \\
\text { and also the methods currently being } \\
\text { used in the analysis. For example, in } \\
\text { X-Ray CT we have moved from rather } \\
\text { fuzzy images of particles defined by } \\
\text { just a few voxels, for which it was even } \\
\text { difficult to define a particle, to really } \\
\text { quite accurate representations of the } \\
\text { particle morphology and even their } \\
\text { contacts. }\end{array}$ & $\begin{array}{l}\text { The guidance given in Rule } 5 \text { is governed } \\
\text { by: (i) practicalities, since too small a } \\
\text { subset spacing leads to very } \\
\text { computationally intensive analyses that are } \\
\text { unnecessarily time consuming to run; the } \\
\text { use of heavy overlapping also becomes an } \\
\text { increasingly artificial way of increasing the } \\
\text { number of measurements because the same } \\
\text { 'data' (pixels) are being used to contribute } \\
\text { to separate determinations of displacement; } \\
\text { and (ii) a desire to minimise the 'potential' } \\
\text { impact of any bias in the computed } \\
\text { displacement fields, which as demonstrated } \\
\text { in the paper, can lead to erroneous strain } \\
\text { fields. Rule } 2 \text { is intended as a general guide } \\
\text { to be followed for typical geotechnical } \\
\text { modelling scenarios where a large cross- } \\
\text { sectional area of a model is being } \\
\text { monitored through a transparent window. It } \\
\text { is concerned predominantly with the } \\
\text { minimum seeding particle diameter } \\
\text { required for robust sub-pixel measurements } \\
\text { to be achieved. Too few pixels per particle } \\
\text { leads to 'peak-locking' errors. In contrast, } \\
\text { too many pixels per particle is potentially } \\
\text { problematic only if the surface of the } \\
\text { seeding particles is of low contrast (i.e. the }\end{array}$ \\
\hline
\end{tabular}




\begin{tabular}{|c|c|}
\hline & $\begin{array}{l}\text { particles are of uniform colouring) since } \\
\text { the effect of camera sensor noise on the } \\
\text { correlations will be amplified. This point is } \\
\text { made clear by the statement that: "...more } \\
\text { pixels will increase the noise unless the } \\
\text { observed surfaces show adequate } \\
\text { contrast." If the seeding particles show } \\
\text { reasonable contrast across the surface of } \\
\text { each particle we see no disadvantage from } \\
\text { having more particles per pixel than the } \\
\text { minimum values given as guidance by Rule } \\
2 \text { and we feel that the current text in Rule } 2 \\
\text { already reflects this. }\end{array}$ \\
\hline $\begin{array}{l}\text { Won't this type of improvement happen } \\
\text { in PIV analyses and the images used for } \\
\text { them? And when that happens will we } \\
\text { still be trying to identify the "centre" of } \\
\text { particles with the current interpolation } \\
\text { methods? At the very least, if I do have } \\
\text { too much information for the current } \\
\text { technology or noise levels, for example } \\
\text { for the number of pixels per particle, I } \\
\text { could simply recombine my pixels to } \\
\text { create larger groups. }\end{array}$ & $\begin{array}{l}\text { Potentially similar improvements in image } \\
\text { capture resolution will probably occur for } \\
\text { the digital cameras used in PIV/DIC } \\
\text { analyses. Experimentalists will likely then } \\
\text { be able to choose between capturing higher } \\
\text { resolution images over the same region of } \\
\text { interest, or the same resolution images over } \\
\text { a larger region of interest. In either case, } \\
\text { we feel that Rules } 2 \text { and } 4 \text {, when used in } \\
\text { combination, are appropriate in guiding } \\
\text { their choice of seeding particle and subset } \\
\text { size. }\end{array}$ \\
\hline $\begin{array}{l}\text { On Fig. } 9 \text { as the particle to pixel size } \\
\text { ratio increased, shouldn't the images of } \\
\text { the particles tend to get sharper? They } \\
\text { seemed to just get larger but retained } \\
\text { the same degree of indistinctness. }\end{array}$ & $\begin{array}{l}\text { In Figure } 9 \text { it is the particles themselves } \\
\text { that are being enlarged; the images are all } \\
1001 \text { by } 1001 \text { pixels in size. The fact that } \\
\text { each particle is represented by a Gaussian } \\
\text { distribution causes the larger particles not } \\
\text { to appear more distinct at their extremities } \\
\text { since the pixel intensity across the particle } \\
\text { is gradually changing with respect to the } \\
\text { radius of the pixel from the centre of the } \\
\text { particle. }\end{array}$ \\
\hline
\end{tabular}


Reviewer 2:

\begin{tabular}{|c|c|}
\hline Comment & Actions \\
\hline $\begin{array}{l}\text { Manuscript COGE-D-15-00327 entitled } \\
\text { "Vermiculate artefacts in image } \\
\text { analysis of granular materials" provides } \\
\text { a cautionary tale of blindly using black- } \\
\text { box measurement tools such as digital } \\
\text { image correlation (DIC) in the absence } \\
\text { of fully appreciating the intricacies of } \\
\text { the underlying working principles } \\
\text { behind the method. In this case, the } \\
\text { paper provides an extremely useful case } \\
\text { study of how poor image texture can } \\
\text { lead to completely erroneous strain } \\
\text { measurements. The paper is of utmost } \\
\text { importance and needs to be published. }\end{array}$ & N/A \\
\hline $\begin{array}{l}\text { Page 1. "Example experimental setups } \\
\text { have been described for element testing } \\
\text { (Rechenmacher \& Finno, 2004; } \\
\text { Bhandari et al., 2012), large scale 1g } \\
\text { testing (White \& Bolton, 2004) and } \\
\text { centrifuge testing (Stanier \& White, } \\
\text { 2013)." While it is understandable the } \\
\text { authors naturally promote their own } \\
\text { work, a considerable body of DIC work } \\
\text { was conducted on the centrifuge before } \\
\text { 2013. Given the early 2000's references } \\
\text { included in the other examples, this } \\
\text { jumps out as appearing somewhat odd. } \\
\text { To avoid the appearance of over- } \\
\text { cheerleading their own work, the } \\
\text { authors are encouraged to reword this } \\
\text { to make the references introduced more } \\
\text { symmetrically between element testing, } \\
\text { 1g, and centrifuge applications. }\end{array}$ & $\begin{array}{l}\text { We appreciate the reviewers concern with } \\
\text { respect to the referencing in this section. To } \\
\text { alleviate the concerns an additional } \\
\text { sentence referencing a number of example } \\
\text { applications of PIV/DIC techniques in } \\
\text { geotechnical modelling investigations has } \\
\text { been added to provide balance in the } \\
\text { referencing. }\end{array}$ \\
\hline $\begin{array}{l}\text { Page 2. "...(ii) the image acquisition } \\
\text { (sensor, lens, illumination, field of } \\
\text { view, image spacing - time or } \\
\text { displacement)". Encourage rewording } \\
\text { to the "image formation process", or if } \\
\text { acquisition is needed, "the image } \\
\text { acquisition process". Encourage } \\
\text { rewording of "image spacing - time or } \\
\text { displacement" as this is awkward and } \\
\text { unclear. }\end{array}$ & $\begin{array}{l}\text { This sentence has been reworded to read } \\
\text { 'image acquisition process (sensor, lens, } \\
\text { illumination, field of view, image capture } \\
\text { frequency)'. }\end{array}$ \\
\hline $\begin{array}{l}\text { Page 2. The paper is generally very } \\
\text { well written. However, the flow of } \\
\text { logic in how the manuscript has been } \\
\text { structured only makes sense once the }\end{array}$ & $\begin{array}{l}\text { Two extra sentences have been added to the } \\
\text { third paragraph of the first section of the } \\
\text { paper. These sentences introduce the } \\
\text { references that provided the impetus for the }\end{array}$ \\
\hline
\end{tabular}


full paper is written. In particular, readers are only really informed on Page 15 in section 5 that the motivation is to attempt to debunk the vermiculation seen in previous experimental studies. At a minimum, a paragraph at the end of Section 1 needs to paint the picture of WHY the work was conducted and to state the objectives of the paper.

Figure 4. This figure states "after Raffel et al." Is this a copied version of their figure (in which case the permissions for duplication need to be addressed), or did you replicate the same type of figure yourselves? If the latter, consider rewording the "after Raffel et al."

Page 5. "More sophisticated sub-pixel displacement refinement can be achieved by incorporating a higherorder subset shape function that allows the displacements within the subset to vary linearly or non-linearly for firstand second-order shape functions respectively." Encourage rewriting this statement to state that "More sophisticated sub-pixel displacement refinement can be achieved by incorporating more complex basis spline curve-fitting of the interpolation peak (e.g. You could include many references here including Sutton et al, Hoult et al., Lee et al, etc, etc) or incorporating a higher-order subset shape function that allows the displacements within the subset to ...." work presented in this paper and read as follows:

"Muir Wood and Lesnieswka (2012) and Nazhat and Airey (2015) provide examples of analyses that have potentially been affected by erroneous numerical artefacts that appear in the strain fields computed from the displacements as highly concentrated bands of shearing. Such features are difficult to reconcile with the mechanics of deformation of granular matter."

The aim of the paper is now clearly stated immediately after as:

"This paper aims to provide guidance on the selection of the most influential experimental parameters in order to avoid spurious features and presents some examples of features that might arise for ill-chosen conditions."

We replicated the same type of figure so we have removed the reference from the figure caption.

We have added the suggested comment about more complex basis spline curvefitting providing an alternative to the nonzero order subset shape function method that is introduced in this section.

Page 8. Figure 5 is referred to in the The figure references have been re-ordered. 
text before figures 3 and 4 . Figures should be reshuffled or text modified.

Page 8. "For geotechnical purposes it is recommended that each subset should contain a minimum of 10 grains in each direction (so that the total number of grains, $n>100$ ), so as to increase the likelihood that the real displacement of the subset of soil can be extracted." This is written very sand-specifically. Perhaps rewrite to make this applicable to either visible soil grains or to flock applied to clay specimens?

Page 9. I must admit that I was somewhat surprised by the $\mathrm{dx}<0.25 \mathrm{Ls}$ restriction. Surely this is more a function of vol. or shear strain rate rather than translation as no loss in correlation strength would be achieved if this tolerance is exceeded. I encourage this rule to either be significantly shortened or removed entirely.

Page 9. In certain parts of the manuscript, the text is somewhat repetitive and wordy. The first paragraph of Section 4 is one location where repetitive items such as retelling of previous section findings and reminding your readers of the definition of image texture could be removed without any loss of clarity.

Figure 9. The lines showing the location of the inset subset are only visible in part a) of the figure. This gets even harder to distinguish once printed in black and white.

Page 11. Please provide equation of Gaussian dots

Rule three: I encourage the authors to place less prominence to the ASR concept as it is a much weaker framework than the MIG or SSSIG. For example, all of the synthetic images in Figure 9 have an ASR $=0.5$, yet performed very differently with regards to peak locking and outcome in terms of error. The Authors have therefore shown that the ASR concept isn't (in itself) predictive of "good texture".

This section of text has been revised so that it is relevant to both sand and clay models as we feel that the general rule given is valid whether dyed sand grains or coloured modelled flock are used to provide the image texture.

On reflection, the authors agree with the reviewer that it is the deformation magnitude that is critical rather than the inplane displacement. Consequently, Rule 6 has been re-written to focus on the deformation magnitude rather than the inplane displacement. References to Rule 6 later in the paper have also been revised.

This section has been tightened up by removing the unnecessarily repetitive text referred to.

This issue has been fixed by thickening the yellow lines.

The equation used is now provided in the appendix.

We accept that it is not ideal that the ASR concept is unable to warn the user of the presence of texture that is susceptible to 'peak-locking'. However, this was not the intention of the $A S R$ measure, which was developed to guide experimentalists in applying an optimal amount of seeding to the exposed face of a geotechnical model. As a result, where the ASR concept is mentioned in the text, the intention of this measure of image texture quality has been 


\begin{tabular}{|c|c|}
\hline $\begin{array}{l}\text { Indeed, in the entire DIC community, } \\
\text { no universal texture measurement has } \\
\text { been fully recognized as "the way } \\
\text { forward" indicative of the complexity } \\
\text { of the topic (the MIG being the closest } \\
\text { to the current front runner). Whereas a } \\
\text { "good ASR" texture with a low d/p } \\
\text { could give a much worse outcome than } \\
\text { a "bad ASR" with higher d/p, the ASR } \\
\text { could be argued to muddy the waters. }\end{array}$ & $\begin{array}{l}\text { clarified. However, we feel that the } \\
\text { criticism of the } A S R \text { measure is perhaps a } \\
\text { little harsh. For instance, take the case } \\
\text { where white seeding particles (sand or } \\
\text { modelling flock) with } d / p \approx 1 \text { are applied to } \\
\text { a black model plane, evenly, until } A S R \approx 0.5 \text {. } \\
\text { All of the } M I G, S S S I G \text { and } \sigma_{I s} \text { measures for } \\
\text { such an image would also indicate high- } \\
\text { quality image texture was present, since the } \\
\text { intensity gradients (and the standard } \\
\text { deviations within the subsets) would be } \\
\text { near maximal. However, as we have } \\
\text { demonstrated, the small seeding particle } \\
\text { diameter would lead to significant 'peak- } \\
\text { locking' for this case. Therefore none of } \\
\text { the proposed measures (MIG, SSSIG, ASR } \\
\text { or } \sigma_{I S} \text { ) are capable of indicating the } \\
\text { likelihood of 'peak-locking' occurring in } \\
\text { isolation and the reviewers criticism of the } \\
A S R \text { measure is equally valid for the other } \\
\text { measures recommended. In all cases the } \\
\text { seeding particle diameter must also be } \\
\text { carefully controlled if 'peak-locking' is to } \\
\text { be avoided. This is why we suggest } \\
\text { checking multiple measures of image } \\
\text { texture quality concurrently, including the } \\
A S R \text {, since no one measure is sufficient to } \\
\text { indicate adequate image texture quality in } \\
\text { isolation. We have now clarified in the } \\
\text { conclusions that the seeding particle } \\
\text { diameter must also be carefully controlled. }\end{array}$ \\
\hline $\begin{array}{l}\text { Page 17. "a different digital camera" - } \\
\text { Higher resolution? Other differences? }\end{array}$ & $\begin{array}{l}\text { The cameras used in the two experiments } \\
\text { reported were very similar (almost identical } \\
\text { resolution in fact). We believe that the } \\
\text { unannealed state of the glass beads in the } \\
\text { latter experiment predominantly led to the } \\
\text { improvement in image texture. This has } \\
\text { now been clarified in an additional footnote } \\
\text { to avoid confusion. }\end{array}$ \\
\hline $\begin{array}{l}\text { Figure } 15 \text { caption. "No vermiculation } \\
\text { present" is perhaps too strong. Consider } \\
\text { applying the same wording in the text } \\
\text { placed in the frame of Figure } 15 \mathrm{~b} \text { ? }\end{array}$ & $\begin{array}{l}\text { We agree. The word 'no' has been replaced } \\
\text { with 'minimal'. }\end{array}$ \\
\hline $\begin{array}{l}\text { In summary, as noted in my } \\
\text { introductory remarks, these comments } \\
\text { are exceedingly minor in nature and the } \\
\text { paper is of very high quality and } \\
\text { significance to the physical modelling } \\
\text { community. I therefore enthusiastically } \\
\text { recommend that it be accepted after }\end{array}$ & N/A \\
\hline
\end{tabular}


these very minor comments have been

addressed.

Reviewer 3:

\begin{tabular}{|c|c|}
\hline Comment & Actions \\
\hline $\begin{array}{l}\text { This manuscript aims to provide } \\
\text { guidance for users of image correlation } \\
\text { techniques in order to avoid unwanted } \\
\text { artefacts from the image processing. }\end{array}$ & N/A \\
\hline $\begin{array}{l}\text { While the work is useful for the } \\
\text { increasing community using PIV/DIC } \\
\text { techniques there are few points that } \\
\text { require further clarification: }\end{array}$ & \\
\hline $\begin{array}{l}\text { Is the discussion limited to a } 2 \mathrm{D} \\
\text { analysis? }\end{array}$ & $\begin{array}{l}\text { The discussion in the paper focusses on the } \\
\text { 2D case where a plane of the model is } \\
\text { exposed to the camera through a } \\
\text { transparent strongbox sidewall. A 3D } \\
\text { PIV/DIC analysis is generally achieved by } \\
\text { performing parallel analyses on multiple } \\
\text { digital images captured from different } \\
\text { perspectives relative to the model, with } \\
\text { parallel interpretation of the displacement } \\
\text { of concurrent particles in each of the } \\
\text { images captured (see the VIC-3D system } \\
\text { for example). Therefore, the discussion } \\
\text { regarding the appropriate size of seeding } \\
\text { particles relative to a single pixel in the } \\
\text { captured images is probably also valid for } \\
\text { 3D PIV/DIC measurements. }\end{array}$ \\
\hline $\begin{array}{l}\text { In section } 3 \text {, for rule } 1 \text { it is stated that a } \\
\text { CCD sensor should be used rather than } \\
\text { a CMOS and the reference Stanier \& } \\
\text { White } 2013 \text { is used. How updated and } \\
\text { informed is this statement? The } \\
\text { reviewer is aware that precision greater } \\
\text { than } 10 \text { microns can be achieved using } \\
\text { a CMOS sensor, can the authors } \\
\text { comment on this? }\end{array}$ & $\begin{array}{l}\text { The differences between CMOS and CCD } \\
\text { sensors outlined in Stanier and White } \\
\text { (2013) is still valid. CCD sensors typically } \\
\text { use a charge to voltage converter; CMOS } \\
\text { sensors generally have one per pixel. Thus } \\
\text { CMOS sensor derived images are less } \\
\text { uniform than CCD derived counterparts } \\
\text { due to variability in the characteristics of } \\
\text { the circuitry performing the charge-to- } \\
\text { voltage conversion. The additional circuitry } \\
\text { utilised in a CMOS sensor typically results } \\
\text { in less of the surface area of the sensor } \\
\text { being used for light capture, leading to } \\
\text { increased signal amplification (gain) } \\
\text { requirements. However, due to } \\
\text { parallelisation of the charge-to-voltage } \\
\text { conversion this process is generally faster } \\
\text { than on a CCD sensor allowing for faster } \\
\text { frame rates. For high quality PIV/DIC }\end{array}$ \\
\hline
\end{tabular}




\begin{tabular}{|c|c|}
\hline & $\begin{array}{l}\text { analyses at moderate frame rates we } \\
\text { recommend CCD sensors for the above } \\
\text { reasons (as outlined in Stanier and White, } \\
\text { 2013). }\end{array}$ \\
\hline $\begin{array}{l}\text { In section } 5, \text { the use of PIV/DIC } \\
\text { techniques for photoelastic material is } \\
\text { debatable as the texture of this material } \\
\text { will change with the mechanical state. } \\
\text { Can the authors comment on this? }\end{array}$ & $\begin{array}{l}\text { The mention of photo-elasticity was } \\
\text { included to provide some context for the } \\
\text { tests reported by Lesniewska and Muir } \\
\text { Wood on assemblies of glass beads. } \\
\text { Photoelastic effects are only seen when the } \\
\text { assembly is viewed with polarised light. } \\
\text { The PIV analyses were performed with } \\
\text { images taken with unpolarised light. } \\
\text { We agree that any change in texture } \\
\text { resulting from deformation in the granular } \\
\text { assembly needs to be small for image } \\
\text { correlation to work. In fact, using light } \\
\text { transmitted through the multiple layers of } \\
\text { grains forming the sample, the deduced } \\
\text { fields of deformation are less influenced by } \\
\text { wall friction effects or by the out of plane } \\
\text { movement (and possible disappearance) of } \\
\text { particles visible in surface observation. }\end{array}$ \\
\hline $\begin{array}{l}\text { The unpublished reference Stanier et al } \\
2015 \text { is cited a number of times in the } \\
\text { paper as an important source of } \\
\text { information and I am wondering if the } \\
\text { manuscript under review shouldn't } \\
\text { actually provide the relevant } \\
\text { information in itself. }\end{array}$ & $\begin{array}{l}\text { The unpublished paper referred to contains } \\
\text { detailed descriptions of the programming } \\
\text { logic and several performance } \\
\text { benchmarking analyses for a recently } \\
\text { developed PIV/DIC algorithm for } \\
\text { geotechnical applications. The manuscript } \\
\text { is still under review; however, it has been } \\
\text { accepted subject to revisions (which have } \\
\text { already been re-submitted to Canadian } \\
\text { Geotechnical Journal) so it will hopefully } \\
\text { be published in the near-future. For this } \\
\text { reason it would be inappropriate to } \\
\text { duplicate the relevant information referred } \\
\text { to in this paper by reference. By the time } \\
\text { the present paper is accepted for } \\
\text { publication we anticipate that the other } \\
\text { reference will be citable more precisely. }\end{array}$ \\
\hline $\begin{array}{l}\text { In the case of acceptance is the } \\
\text { manuscript going to be produced using } \\
\text { coloured figures? This is an important } \\
\text { issue since the b/w figures are not } \\
\text { readable. }\end{array}$ & $\begin{array}{l}\text { Should the manuscript be accepted we will } \\
\text { consider publication with colour figures, if } \\
\text { required by the journal editors. }\end{array}$ \\
\hline
\end{tabular}




\title{
Vermiculate artefacts in image analysis of granular materials
}

\author{
Sam Stanier ${ }^{1}$, Jelke Dijkstra ${ }^{2}$, Danuta Leśniewska ${ }^{3}$ \\ James Hambleton ${ }^{4}$, David White ${ }^{1}$, David Muir Wood $^{5,2,1}$ \\ ${ }^{1}$ Centre for Offshore Foundation Systems, University of Western Australia \\ ${ }^{2}$ Division of Geo-engineering, Chalmers Tekniska Högskola, Göteborg, Sweden \\ ${ }^{3}$ Politechnika Koszalińska, Koszalin, Poland \\ ${ }^{4}$ Department of Civil Engineering, Newcastle University, New South Wales \\ ${ }^{5}$ Division of Civil Engineering, University of Dundee, United Kingdom
}

\begin{abstract}
Some reported analyses of images of deforming granular materials have generated surprising vermiculate strain features which are difficult to reconcile with the mechanics of deformation of granular matter. Detailed investigation using synthetic images and improved processing of images of laboratory experiments indicates that such features can emerge as a consequence of the image acquisition (sensor, contrast, resolution), the subsequent image correlation implementation, and the user's choice of processing parameters. The two principal factors are: (i) the texture and resolution of the images and (ii) the algorithm used to achieve sub-pixel displacement resolution. Analysis of the images using a sub-pixel interpolation algorithm that is more robust than that used originally eliminates the vermiculate features for images with moderate resolution and texture. However, erroneous features persist in images with low resolution and poor texture. Guidance is provided on ways in which such artefacts can be avoided through improved experimental and image analysis techniques.
\end{abstract}

Keywords: granular materials, digital image correlation, vermiculation, sub-pixel interpolation

\section{Introduction}

Image analysis has become a widely used tool for obtaining full-field information of displacements in granular media. The process starts with the design of an experiment that allows the acquisition of digital images of the deforming material (e.g. behind a transparent window) at chosen intervals. Example experimental setups have been described for element testing (Bhandari et al., 2012), large scale 1g testing (White \& Bolton, 2004) and centrifuge testing (Stanier \& White, 2013). Similar equipment has been used to capture images to investigate a wide range of geotechnical phenomena, including: the development of shear bands in sands (Rechenmacher \& Finno, 2004); seasonal rainfall-induced slope failures (Take \& Bolton, 2004); the effects of tunnelling in sand on greenfield settlements (Marshall et al. 2012); and fault rupture propagation in sand (Anastasopoulos et al.. 2007). ir 
Several image processing techniques have been developed to deduce displacements from analysis of successive images captured using such experimental apparatus, e.g. image subtraction (Keshavarzy \& Ball, 1999; Rosenbrand \& Dijkstra, 2012), particle tracking (Crocker \& Grier, 1996; Ando et al., 2012) and image correlation techniques such as Particle Image Velocimetry (PIV) (Westerweel, 1997; White et al., 2003) and Digital Image Correlation (DIC) (Sutton et al., 1983). With adequate image texture (the number and contrast of spatial features and/or grains), image correlation techniques are most suitable for obtaining accurate displacement fields with high spatial resolution.

However, it is not trivial to retrieve meaningful displacement data from such experiments. The quality of the image texture (or speckle pattern) is typically dictated by: (i) the natural contrast of coarse grained material or the artificial seeding applied to the surface of the specimen that is visible to the digital camera through a transparent window (which is dependent on the scale or resolution of the image because this determines how well individual grains can be distinguished); (ii) the image acquisition process (sensor, lens, illumination, field of view , image spacing - time or displacementand image capture frequency); and (iii) any image pre-processing applied prior to the displacement computations. In addition, the image correlation algorithm chosen to compute the displacements needs to be carefully selected in order to avoid numerical artefacts in the image analyses. This paper provides-Muir Wood \& Leśniewska (2012) and Nazhat \& Airey (2015) provide examples of analyses that have potentially been affected by erroneous numerical artefacts that appear in the strain fields computed from the displacements as highly concentrated bands of shearing. Such features are difficult to reconcile with the mechanics of deformation of granular matter. This paper aims to provide guidance on the selection of the most influential experimental parameters in order to avoid spurious features and presents some examples of features that might arise for ill-chosen conditions.

\section{Image correlation: a brief description}

The tools employed in geotechnical applications of image analysis have either been developed within the community (White et al., 2003; Hall et al., 2010) or have been borrowed from adjacent disciplines, e.g. open source PIV tools such as MatPIV (Sveen, 2004), PIVlab (Thielicke \& Stamhuis, 2014), OpenPIV (Taylor et al., 2010) and JPIV (Vennemann, 2015). Meanwhile developments in experimental mechanics and fluid mechanics continue (Pan et al., 2013; Scarano, 2013).

The ability to extract displacement fields from image correlation rests on four implicit assumptions (Adrian \& Westerweel, 2011):

- The particles that are observed are homogeneously distributed across the image. This will usually be automatically satisfied for sands, where the individual grains often contain sufficient natural colour variation to provide 


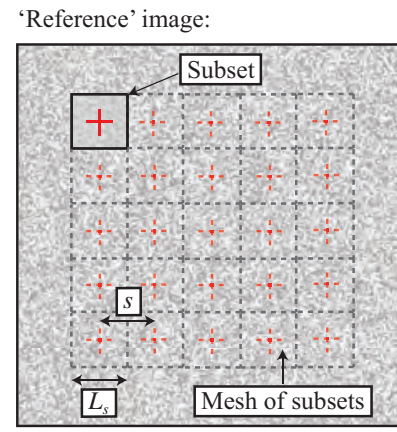

(a)

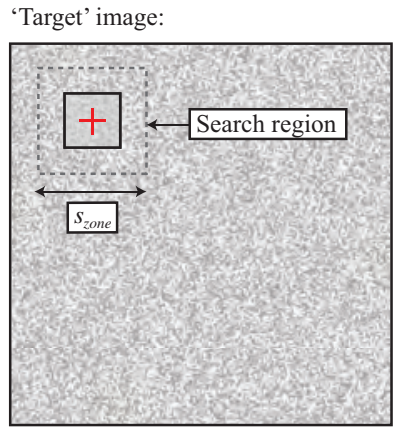

(b)

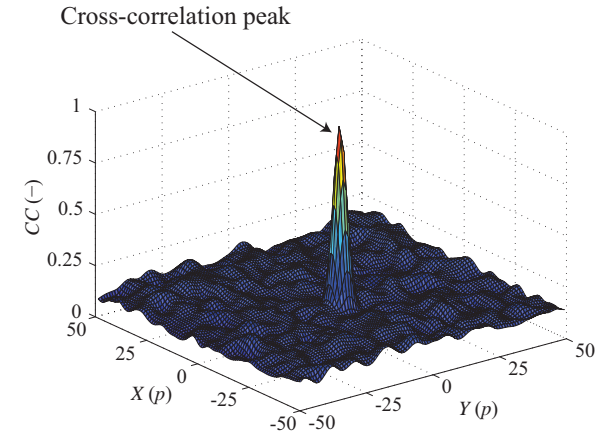

(c)

Fig. 1: Schematic diagram of typical PIV/DIC computations: (a) definition of mesh of subsets on 'reference' image, (b) computation of cross-correlation $(C C)$ over a region encompassing the subset in the 'target' image, and (c) location of the peak in crosscorrelation to the nearest integer pixel values.

adequate contrast. For clay models individual particles are not visible and a (homogeneous) surface speckle has to be added by artificial seeding.

- The observed natural or imposed image texture provides a perfect representation of the displacement of the soil. Wall friction may make the observed displacements unrepresentative of the displacements occurring through the thickness of the material.

- The 'reference' and 'target' images are sufficiently similar (i.e. the deformation is small enough) that a spatial measure of correlation can be computed and a clear peak isolated for all subsets (also known as patches or interrogation windows).

- The shape (or warp) function (which mathematically describes how the subset is allowed to displace and deform during the displacement computation) used by the image correlation algorithm should be consistent with the deformation being measured. Simpler correlation algorithms assume that the transformation from 'reference' to 'target' is a pure translation; more sophisticated algorithms may accept distortion or rotation in addition to translation.

Most freely available PIV/DIC algorithms, including those referenced here, perform two separate computations: (i) selection of a subset within the 'reference' image for which the peak in cross-correlation can be sought in successive 'target' images, for each subset, to the nearest integer pixel coordinates; and (ii) refinement of this measurement by interpolation of the cross-correlation for the subset corresponding to the correlation peak and a selection of its neighbours in order to refine the displacement measurement to sub-pixel resolution. Algorithms which employ a zero-order subset shape function which only permits the subset to be translated when seeking to maximise the cross-correlation cannot accommodate 


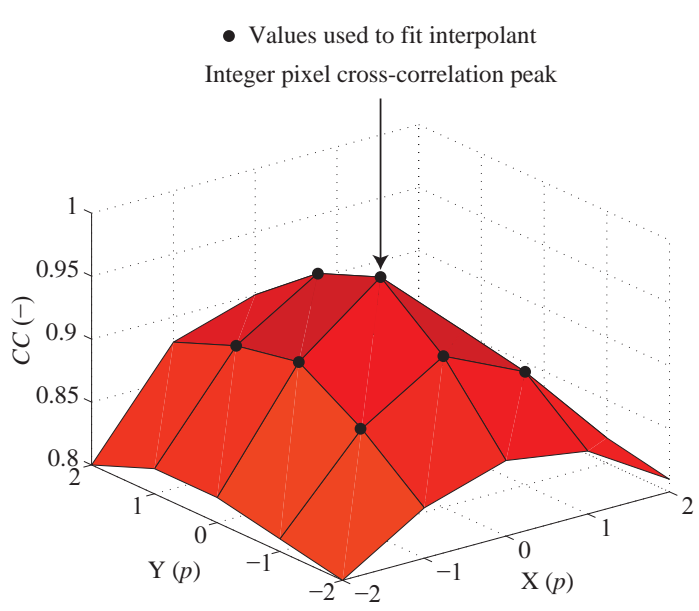

(a)

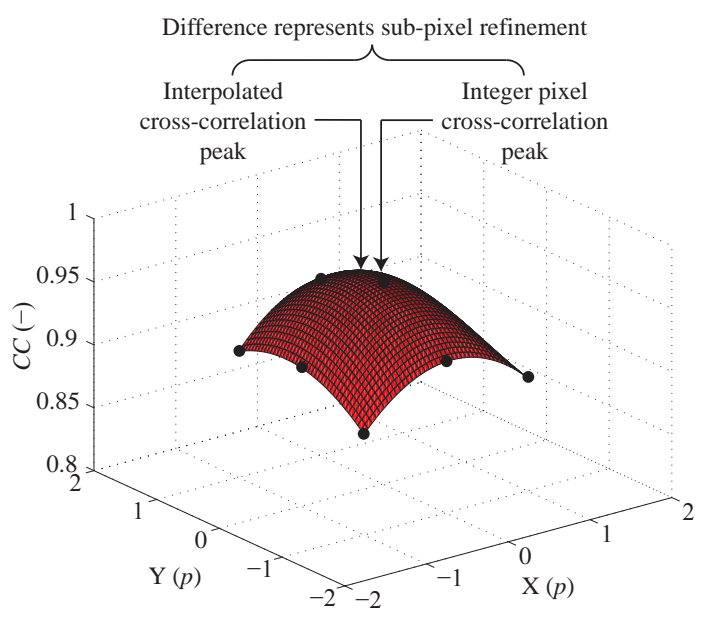

(b)

Fig. 2: Conventional sub-pixel refinement: (a) isolation of the cross-correlation peak and values for neighbouring subsets (at integer pixel locations) and (b) bi-cubic interpolation of the cross-correlation peak and the eight nearest neighbours to identify an interpolated peak to sub-pixel resolution.

significant distortion or rotation. Large gradients of displacement across the subset being interrogated can lead to an inability to correlate 'reference' and 'target' images, inevitably resulting in measurement errors.

\subsection{Cross-correlation of the subsets}

Subsets of an initial 'reference' image are compared with subsequent 'target' images in order to calculate a spatial measure of cross-correlation $(C C)$ (see Fig 1). Two popular measures are 'normalised cross-correlation' $(N C C)$ (Lewis, 1995) and 'zero normalised cross-correlation' $(Z N C C)$ (Pan et al., 2010). The zero normalised cross-correlation coefficient $\left(C C_{Z N C C}\right)$ represents a robust measure of correlation as it can accommodate variations (offset and/or scale) in brightness across the image, with values of 1,0 and -1 indicating perfect, zero and inverse correlation, respectively. Mathematical definitions for $C C_{Z N C C}$ and other measures used throughout this paper are provided in the Appendix.

\section{$1.2 \quad$ Sub-pixel displacement refinement}

The integer displacement estimate from the first step is refined using sub-pixel interpolation functions. Typically bi-cubic splines or Gaussian functions are fitted to the correlation peak and the neighbouring values (Figure 2). The maximum value of the interpolant provides an improved estimate of displacement typically to sub-pixel precision of the order of $0.01 p$ (White et al., 2003), where $p$ is the pixel size. 
More sophisticated sub-pixel displacement refinement can be achieved by incorporating more complex basis spline curve-fitting of the interpolation peak (Lee et al. 2012). Alternatively a higher-order subset shape function that allows the displacements within the subset to vary linearly or non-linearly for firstand second-order shape functions respectively . Such methods ( Yu \& Pan, 2015), could be incorporated. Methods with higher-order subset shape functions tend to deal with spatially varying deformation fields more robustly but require additional image intensity interpolation and optimisation techniques and have until recently not been widely available. Ncorr (Blaber et al., 2015) and GeoPIV-RG (Stanier et al., 2015) are two recently developed examples of PIV/DIC software which incorporate such an enhanced sub-pixel displacement refinement and which are now freely available to the geotechnical research community.

\section{Requirements for accurate cross-correlation}

The following design rules are based on experiences in fluid mechanics using PIV to analyse the trajectories of tracers in fluid flow (e.g. Adrian \& Westerweel 2011), DIC analysis of a speckle pattern on a material surface (e.g. Sutton 2008), and recent experience of performing PIV/DIC analyses on various laboratory geomaterials (e.g. Stanier \& White 2013).

R1 Image quality: A large Signal-to-Noise Ratio $(S N R)$ (see Appendix for definition) using the full dynamic range of the image sensor is desirable. Provide sufficient and uniform illumination of the specimen. Use high quality optics characterised by a small available $f$-stop (absence of optical aberrations); then set aperture with the highest value of $f$-stop (smallest aperture size) compatible with the available illumination and desired depth of field. Select a camera with a global rather than rolling shutter, and with a ChargeCoupled Device (CCD) sensor rather than a Complementary Metal Oxide Semiconductor (CMOS) sensor (Stanier \& White, 2013). Images with a uniform distribution in the intensity histogram with values between 20 and 225 are considered high quality for an 8-bit sensor (maximum 255 intensity levels). Values lower than 20 are often associated with sensor noise, whilst values above 220 are approaching sensor saturation (Sutton, 2008). Image formats with high compression will reduce the $S N R$ of the image (e.g. Cosman et al. 1994). Uneven or fluctuating illumination has a large impact on the subsequent analysis if non-normalised cross-correlation is used (e.g. Tong 2005). Quantitative assessment of global image quality can be provided by checking the Mean Intensity Gradient (MIG) proposed by Pan et al. (2010).

R2 Information in the signal: The spatial resolution of the digital image data, stored in a pixel array, where each pixel holds an intensity value for the amount of light that fell on that pixel, is discrete. Hence, there should be a 


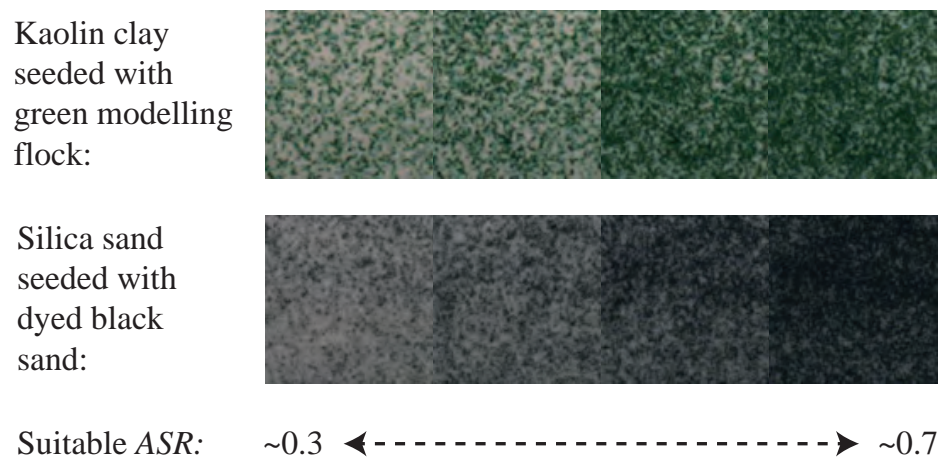

Fig. 3: Examples of suitable Artificial Seeding Ratio $(A S R)$ for Kaolin clay and Silica sand after Stanier \& White (2013).

sufficient number of pixels in the spatial feature that needs to be followed, for example the grain, in order to retain enough information in the signal. Theoretical and experimental analysis from fluid mechanics has shown that this requires 2 pixels across each particle (Westerweel, 2000), implying that for soils $d_{50} / p \sim 2$, where $d_{50}$ is the median grain size. Fewer pixels will not resolve the grain and more pixels will increase the noise unless the observed surfaces of the grains show adequate contrast. In solid mechanics where higher seeding densities (denser speckles) are more common the average recommended feature size is doubled, to $4 \times 4$ pixels. Sutton $(2008)$ introduces a more systematic way of determining the feature size of the speckle in an image by performing autocorrelation and recommends sizes of $3 \times 3$ to $7 \times 7$ pixels.

R3 Texture or speckle quality: The grains, or imposed speckle, should provide sufficient contrast between the particles contained within the subsets. The intensity differences between the grains and the voids should be larger than the noise in the image. In medical imaging this feature is sometimes called contrast resolution (Stelzer, 1998). This requirement is readily satisfied when images with high $S N R$ and materials with distinct colour differences are used. In geotechnical applications other measures of image texture have been recommended and acceptance values proposed (Stanier \& White, 2013). These include Stanier \& White (2013) recommend that seeding be applied to models such that: (i) the Artificial Seeding Ratio is in the range of $0.3<A S R<0.7$ (see Figure 3 ) and so as to ensure an optimal quantity of seeding has been applied to the model; and (ii) the standard deviation of the subset intensities $\sigma_{I_{s}}>\sim 15 \sigma_{I_{\text {s }}}$ is greater than 15 so as to ensure that there is sufficient contrast within the subsets. The Sum of Squares of Subset pixel Intensity Gradients $(S S S I G)$ provides another alternative measure of subset quality with a minimum threshold for precise measurements of $S S S I G>\sim 1 \times 10^{5}$ recommended by (Pan et al., 2008).

R4 Subset size: For geotechnical purposes it is recommended that each subset 


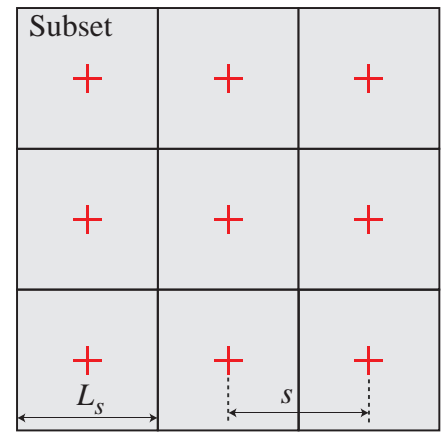

(a)

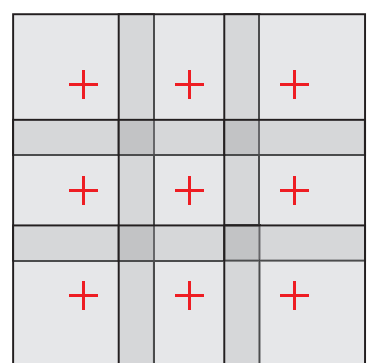

(b)

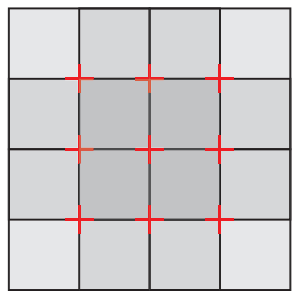

(c)

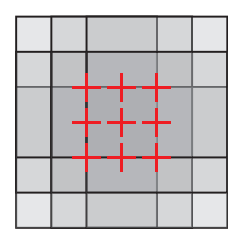

(d)

Fig. 4: Subset of size $L_{s} \times L_{s}$ at spacings of $s$ for overlapping ratios $\left(\left(L_{s}-s\right) / L_{s}\right)$ of: (a) 0.0 , (b) 0.25 , (c) 0.5 and (d) 0.75 . Darker regions represent oversampling caused by increasing overlap, resulting in increased spatial resolution.

should contain a minimum of 10 grains contrasting particles (whether sand grains or artifical seeding such as modelling flock) in each direction (so that the total number of grainsparticles, $n>\sim 100$ ), so as to increase the likelihood that the real displacement of the subset of soil can be extracted. At the same time the subset should be sufficiently small for the order of its shape function to be consistent with the deformation being measured. Higher-order (first- or second-order) shape functions improve correlation of subsets which undergo spatially varying deformations and thus allow the use of larger subsets, which will typically contain more information and provide more robust correlations. In practice, a subset size of $24 \times 24$ to $48 \times 48$ pixels provides a good starting point from which trial and error refinement can be performed if deemed necessary.

R5 Subset overlap: Overlapping of subsets for adjacent points permits more data to be obtained from the image resulting in increased spatial resolution (i.e. over-sampling; see Figure 4). However, bias errors can arise when the subset shape function is incompatible with the deformation of the subset that is being measured (Schreier \& Sutton, 2002). Overlapping can amplify the error in computing strains from the derivatives of the displacement fields (Raffel et al., 2007). In fluid dynamics overlap ratios of 0.2 to 0.5 are recommended as a good compromise between maximising the number of measurement points and minimising the impact of potential bias in the displacement field. Plotting histograms of either the total or sub-pixel displacement component magnitudes provides a ready check for the presence of bias errors. Bias is evident when saw-toothed total displacements or non-uniform sub-pixel displacement distributions are apparent (see Figure 5 and Raffel et al. (2007)): the locking of displacement peaks onto integer pixel values of displacement (Figure $5(\mathrm{a})$ ) is unlikely to be a realistic representation of the actual displacement field. 


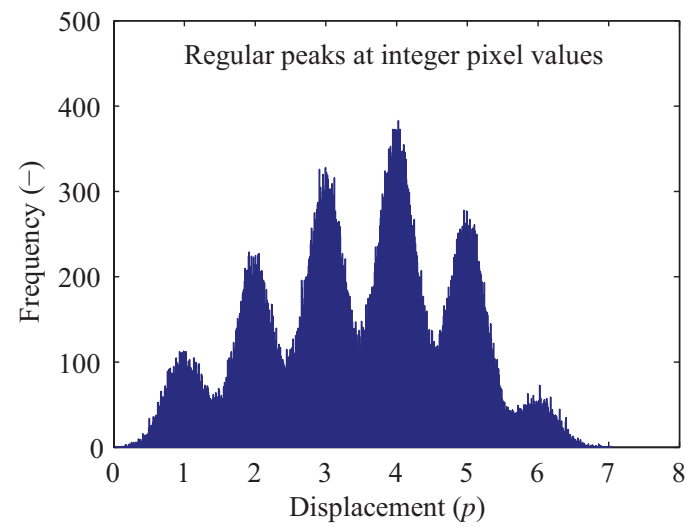

(a)

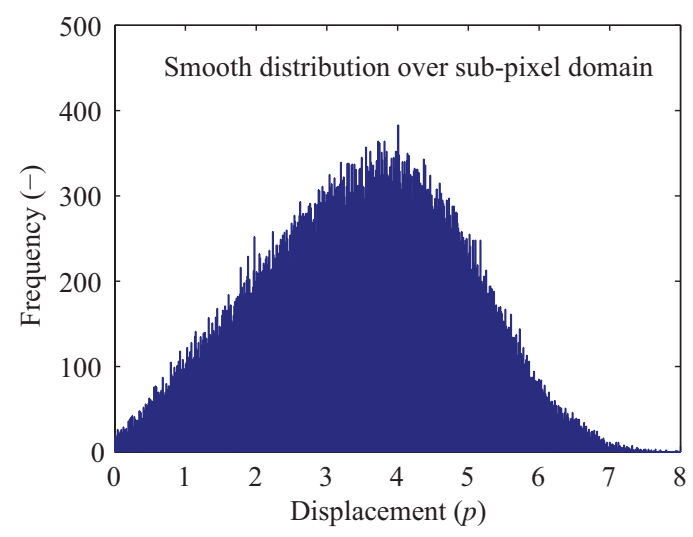

(c)

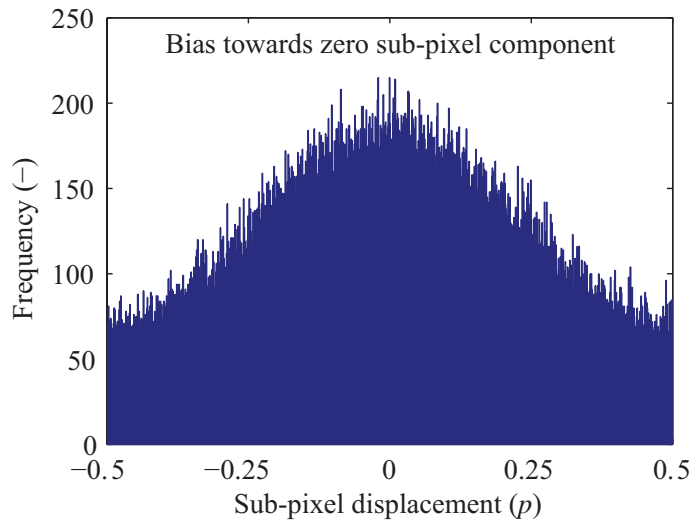

(b)

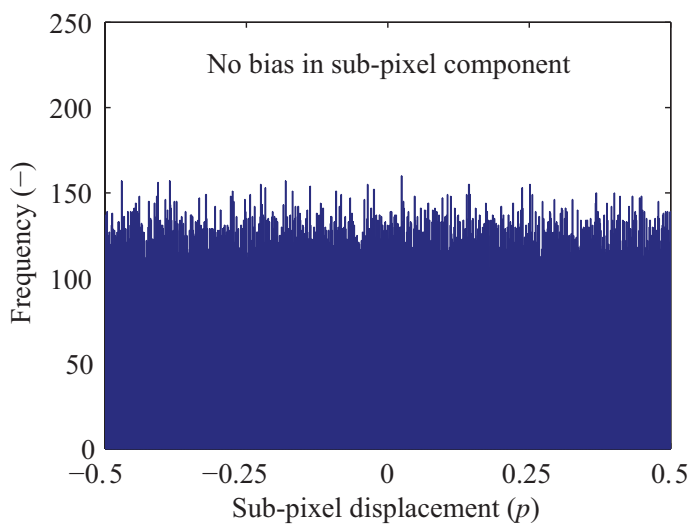

(d)

Fig. 5: Histograms of displacement magnitudes: $(\mathrm{a}, \mathrm{b})$ total and sub-pixel displacement components with bias errors and (c, d) total and sub-pixel displacement components without bias errors.

R6 In-plane motionDeformation magnitude: For fypiealbasic PIV/DIC implementations that process the subsets over successive rows or columns and use interpolation of the correlation peak to achieve sub-pixel displacement refinement, the displacement increment do not allow the subsets to deform (i.e. zero-order subset shape function), the maximum deformation or strain occuring between two cross-correlated images should not be too large, i. e. $|\delta x|<{ }_{4}^{1} L_{s}$, where $L_{s}$ is the size of the subset. . In some of the experimental observations presented later in $\S 4, t_{s}=48$ pixels and the allowed maximum displacement would be 12 pixels $\sim 1.2 \mathrm{~mm}$ displacementrelatively large deformations are observed between successively captured image pairs (engineering shear strains $\chi>30 \%$ ) leading to persistent bias errors. In practice such a limit is required to minimise the deformation experienced within the subset subsets between the 'reference' and 'target' images thereby preserving should be limited to preserve the correlation as well as minimising the area over which the correlation measure is to be computed, which generally results in less computational expense. In fluid mechanics 
where large displacements are more common, window shifting techniques have been developed to overcome these problems (Gui \& Wereley, 2002) This can be achieved by capturing additional images at intermediate intervals. Algorithms that use higher-order subset shape functions (e.g. Blaber et al. 2015; Stanier et al. 2015) and image intensity interpolation and optimisation to achieve sub-pixel measurement resolution can tolerate greater displacements between images and this displacement limit can be somewhat relaxed. Such algorithms tolerate larger displacements and deformations before decorrelation leads to erroneous measurements. Automatic 'reference' image updating schemes that use the correlation coefficients of the subsets to decide when to update the 'reference' image, such as those described by Pan et al. (2012) and Stanier et al. (2015) can be used to process the additional images in an optimised manner. These methods tend to be more computationally intensive for single computations; however, preconditioning of the optimisation problem for each subset by assimilation of information from the neighbouring subset having the best correlation leads to vastly improved efficiency for subsequent computations. It is then unnecessary to calculate the initial measure of correlation over large areas of the 'reference' and 'target' images for every subset.

\section{Spurious features: synthetic data}

In the previous section bias - locking of computed displacements onto integer pixel values - was identified as a potential source of error. The following synthetic analyses demonstrate that numerical artefacts can appear in PIV/DIC analyses of geomaterial if the particles or features that make up the image texture (the changes in image brightness resulting from the speckles or grains that make up the image features) are too small relative to the pixel size in the images captured (contravening rule R3). In this instance- causing the sub-pixel displacements become undetectable and the sub-pixel refinement stage (Section 1.2) of the analysis breaks down.

To investigate the detectability of sub-pixel displacements, synthetic-refinement process to break down. Synthetic images containing single particles of different sizes were generated, displaced by sub-pixel distances and subsequently analysed using zero-order PIV/DIC techniques. The synthetic images were $25 \times$ 25 pixels in size, with a black background onto which white dots with varying brightness of Gaussian distribution were projected to sub-pixel precision. The intensities of each pixel in the images were estimated from the Gaussian eurves distribution describing the brightness of the dot (see the Appendix for a mathematical definition). Figure 6 shows the four initial images generated by this process for dot sizes, $d=p, 2 p, 4 p$ and $8 p$. The dot in the 'reference' image was then displaced horizontally in increments $\delta x=0.02 p$ over the range of $-0.5 p<\delta x<0.5 p$. The zero normalised cross-correlation coefficient $\left(C C_{Z N C C}\right)$ was calculated for a centrally located $15 \times 15$ pixel subset and the eight neigh- 
$d / p=1:$

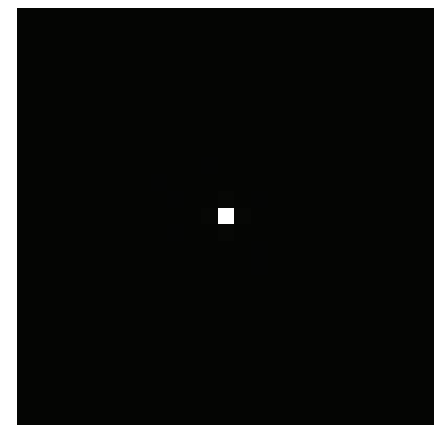

$d / p=4$

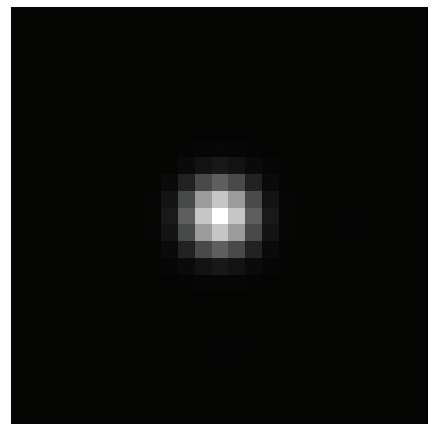

$d / p=2:$

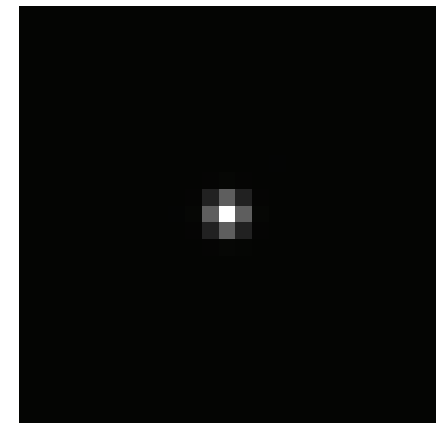

$d / p=8:$

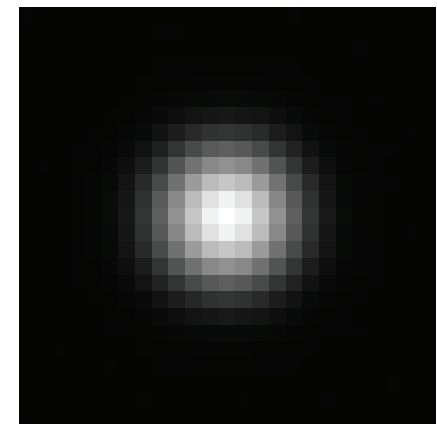

Fig. 6: Example images for the single particle displacement analysis with particle/pixel size ratios: (a) $d / p=1$; (b) $d / p=2$; (c) $d / p=4$; and (d) $d / p=8$.

bouring subsets using Equation (1)(see Appendix).

A bi-cubic interpolant was fitted to the correlation surface described by the $C C_{Z N C C}$ values of the subsets and sampled at intervals of 0.005 pixels (i.e. the smallest non-zero displacement that could possibly be measured was 0.005 pixels). The location of the peak of the interpolant, which provides the best estimate of the displacement to sub-pixel resolution, is compared with the imposed displacement in Figure 7 for particle/pixel size ratio $d / p=1,2,4$ and 8 . Figure 8 presents cross sections of the interpolated correlation peak at displacement increments, $\delta x=-0.4 p, 0$ and $0.3 p$ with the inferred and imposed displacements indicated by dashed and solid lines respectively.

When $d / p$ is small, there is a bias in the computed displacements towards the integer pixel value closest to the correlation peak (zero in the present example), and the inferred and imposed displacements diverge for non-zero displacements (Figure 7). The interpolant has a sharp peak because the neighbouring subsets have very poor correlation by comparison with this centrally located peak correlation (Figure 8). This phenomenon is known as 'peak locking' (Westerweel, 1997). As $d / p$ increases the correlation in the neighbouring subsets improves causing the interpolated correlation peak to shift location. As a result, the bias reduces and the displacements inferred are much closer to those imposed. However, if 


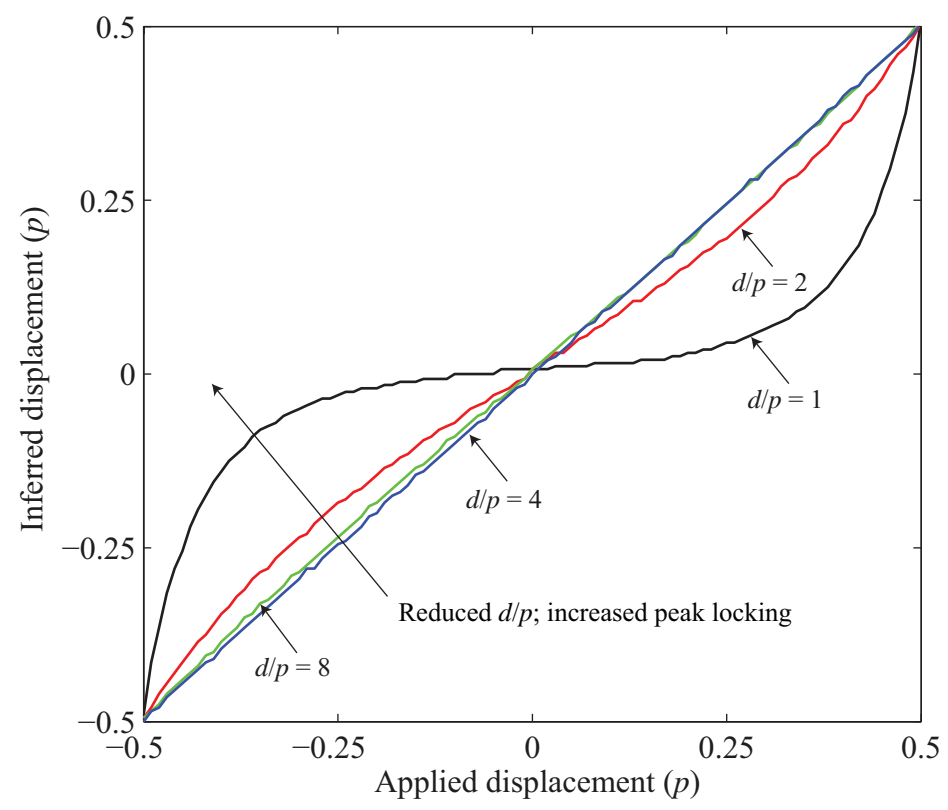

Fig. 7: Impact of particle/pixel size ratio, $d / p$, on the difference between inferred and actual displacement.

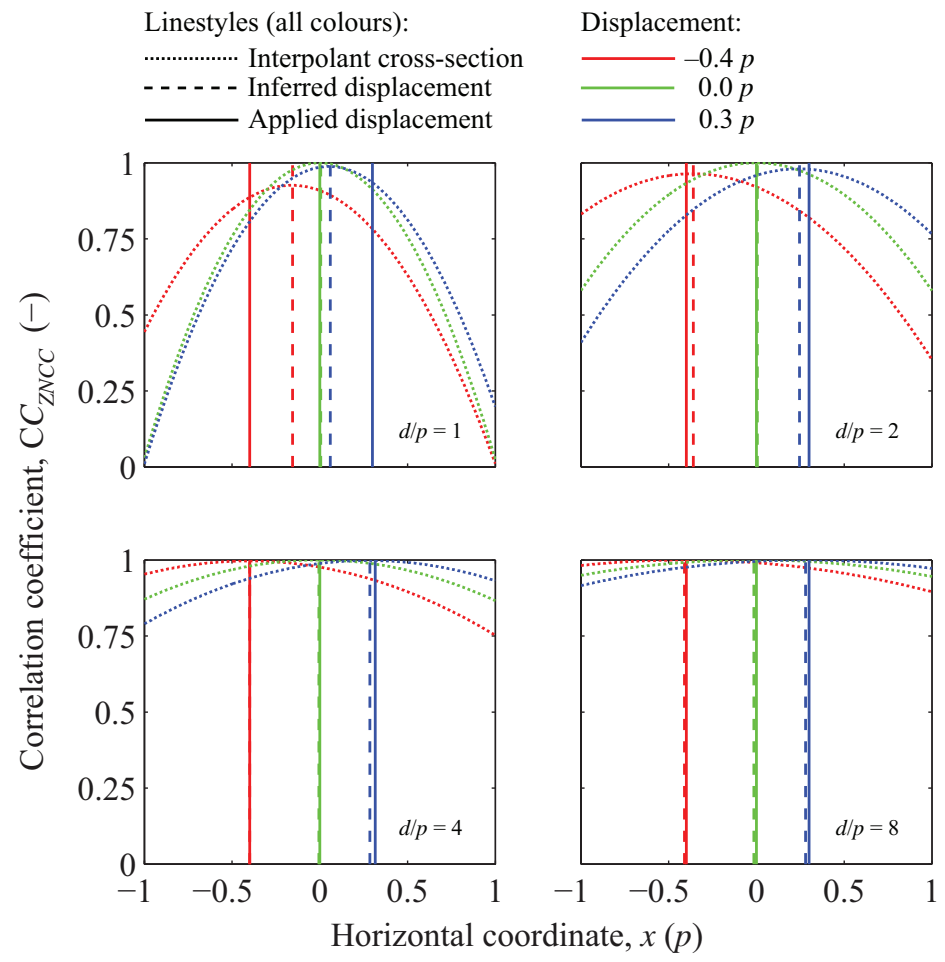

Fig. 8: Impact of particle/pixel size ratio, $d / p$, on the shape and bias of the bi-cubic interpolant fitted to the correlation coefficient $\left(C C_{Z N C C}\right)$ peak and its eight nearest neighbours to achieve sub-pixel measurement resolution. 
the grains are of uniform colour, and have a high $d / p$ ratio, (so that there are several pixels in a single particle), then undesirable 'correlation noise' can develop with many spurious peaks (see rule R3). We can imagine three cases: with small $d / p$ the cross-correlation function has a sharp peak which cannot be refined by sub-pixel interpolation; with large $d / p$ but with grains which lack contrast the correlation function is noisy; with large $d / p$ and with contrasting grain texture the correlation should be optimal.

In order to demonstrate the impact that 'peak locking' can have on strain field computations involving geomaterials, an artificial smooth displacement field was imposed on synthetic soil-like images. The initial 'reference' images were generated by randomly projecting thousands of white dots - each with intensity described numerically by a Gaussian curve (see the Appendix for a mathematical definition), as in the previous section - onto a black background. Sufficient dots were projected until each image had a mean pixel intensity of $\sim 125$ (for a gray scale image intensity ranging from 0 to 255). This ensured that each image had the same amount of pixel information, but the images were arranged in different ways. In each analysis the dots were all of the same size, but analyses were run with dot diameters between 1 and 10 pixels in 0.25 pixel increments. Figure 9 shows four of the images generated with particle to pixel size ratios $d / p=1,2$, 4 and 8 respectively. A Flamant displacement field (Flamant, 1892) was then imposed onto the 'reference' positions of the dots to create a 'target' image for each value of $d / p$ (Figure 10). This smoothly varying displacement field contains both supra- and sub-pixel displacement magnitudes and implies correspondingly smooth variations of engineering shear strain, $\gamma$.

The displacement fields between each pair of images were analysed using both GeoPIV and the first-order subset deformation variant GeoPIV-RG (Stanier et al., 2015). The analysis control parameters adopted for both algorithms are given in Table 1. Figure 11 presents the fields of engineering shear strain, $\gamma$, for particle size ratios $d / p=1,2,4$ and 8 computed using GeoPIV and GeoPIV-RG. Spurious features are clearly evident for the GeoPIV analyses for the smallest particles but these disappear as the particle size $d / p$ increases. Examination of the displacement fields reveals that these features are always located where the true displacement is a half-integer value $\delta x=m p / 2(m \in \mathbb{Z})$. The interpolation process has failed and the sub-pixel interpolation step essentially recovers the nearest integer pixel value (cf. Figure 7). As the particle size rises to $d / p=8$ the spurious features largely disappear, but the strain fields have noise of $\sim 1 \%$. The GeoPIV-RG analyses only show the spurious features for $d / p<2$ (the theoretical limit derived by Westerweel (2000)), but these are less pronounced than for the GeoPIV analysis. Figure 12 shows that the optimal correlation coefficient $\left(C C_{Z N C C}\right)$ output by GeoPIV-RG is much higher than that computed for the zero-order subset deformation analyses of GeoPIV, where $C C_{Z N C C}$ degrades as the deformation magnitude rises. The improved algorithms are significantly less susceptible to 'peak locking' and much more precise. 


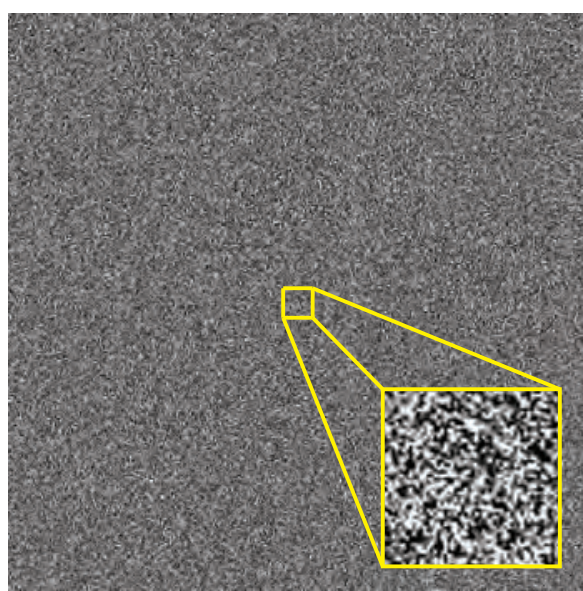

(a)

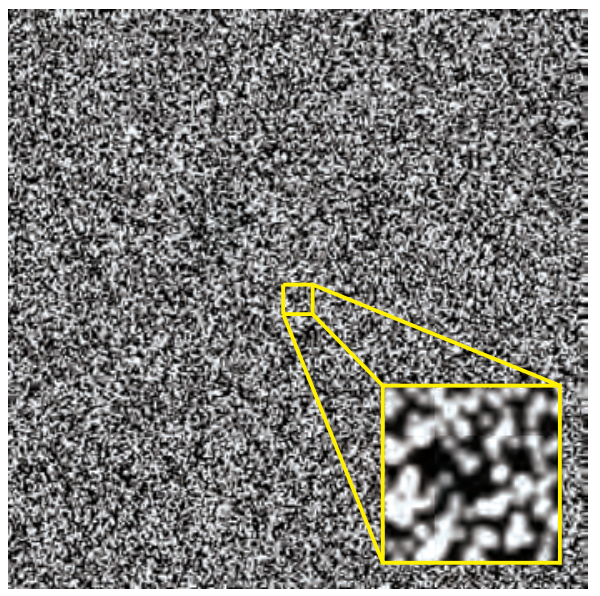

(c)

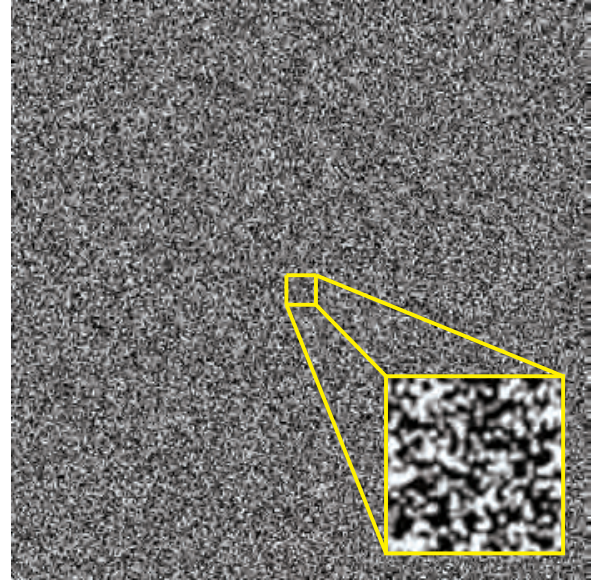

(b)

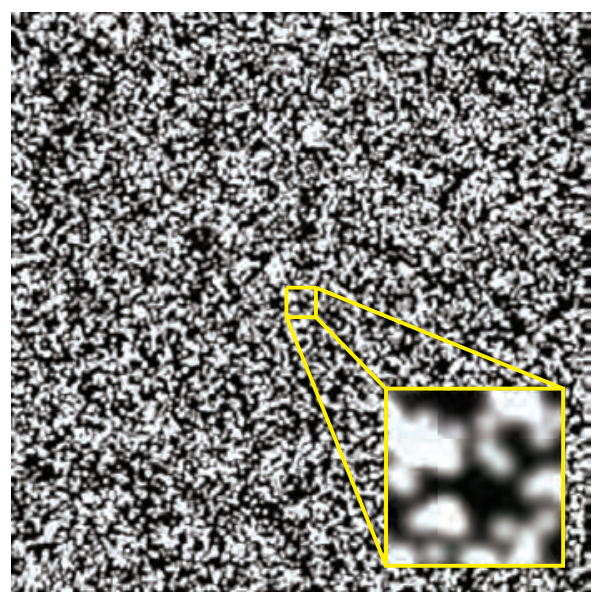

(d)

Fig. 9: Randomly generated synthetic images with $A S R \sim 0.5$ (Stanier \& White, 2013) with zoomed inset of approximate subset size $(45 \times 45$ pixels $)$ highlighted: particle/pixel size ratios: (a) $d / p=1$; (b) $d / p=2$; (c) $d / p=4$; and (d) $d / p=8$.

\section{Spurious features: real data}

Reporting of surprising vermiculate strain features in model tests described by Muir Wood \& Leśniewska (2012) and by Nazhat \& Airey (2015) provided the initial impetus for the studies reported in this paper. Leśniewska \& Muir Wood (2009) observed glass ballotini, of $1 \mathrm{~mm}$ diameter, contained in a box approximately $200 \times 200 \times 20 \mathrm{~mm}$. A series of articulated footings applied a controlled stress to the surface of the granular material (Figure 13). The material was retained by a wall whose outward translation could be controlled, producing deliberate rotation of principal axes of strain. The particles were immersed in a fluid with closely matched refractive index so that when observed with polarised light the photoelastic properties of the glass particles could be exploited to obtain information connected to the stress state in the granular material. The texture 


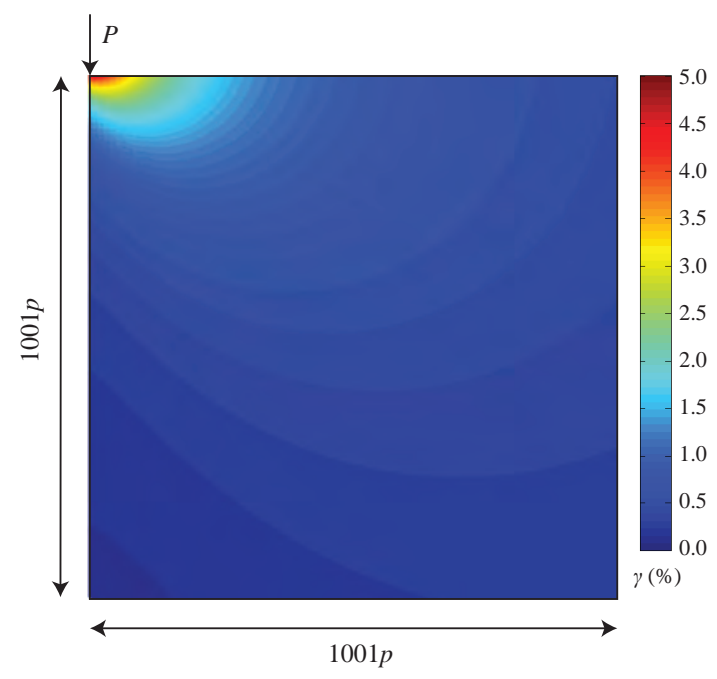

Fig. 10: Engineering shear strain field, $\gamma$, generated for a Flamant displacement field with an arbitrary value for the point force, $P$.

Table 1: GeoPIV and GeoPIV-RG analysis settings

\begin{tabular}{lc} 
GeoPIV $^{*}$ & \\
\hline$L_{s}$ & $48 p \times 48 p^{\ddagger}$ \\
$s$ & $12 p$ \\
$s_{\text {zone }}$ & $15 p$ \\
& \\
GeoPIV-RG & \\
\hline$D_{s}$ & $54 p^{\ddagger}$ \\
$s$ & $12 p$ \\
$m a x_{\text {iter }}$ & 50 \\
$|\Delta p|_{\text {max }}$ & $1 \times 10^{-5}$ \\
$C C_{\text {ZNCC-seed-tol }}$ & 0.9 \\
$C C_{Z N C C-\text { max-tol }}$ & 0.75
\end{tabular}

* $s_{z o n e}$ is the search zone over which the cross-correlation function is estimated for each subset, as illustrated in Figure 1 and described by White et al. (2003).

$\dagger$ max $_{\text {iter }}$ is the maximum number of deformation parameter optimisation iterations; $|\Delta p|_{\text {max }}$ is the norm of the warp function difference which is used as an exit criterion for the deformation parameter optimisation process with small values indicating that an optimised solution has been found; $C C_{Z N C C-\text { seed-tol }}$ is the tolerance on the correlation coefficient for the seed computation and $C C_{Z N C C-\max -t o l}$ is the tolerance on the correlation coefficient for the full displacement field. See Stanier et al. (2015) for further details.

$\ddagger$ Subset sizes chosen to have near equivalent area (within $\sim 0.6 \%$ ). 


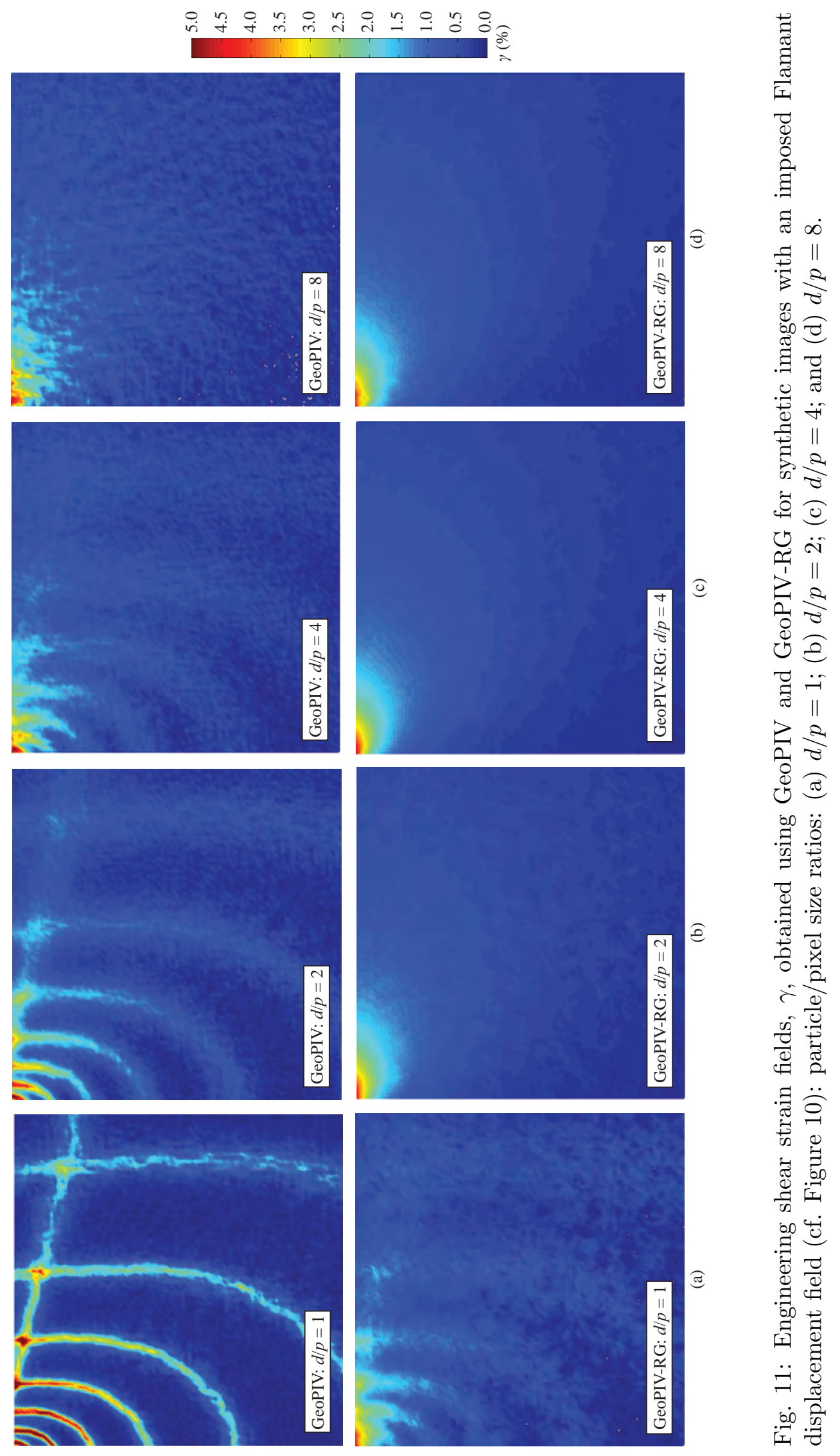




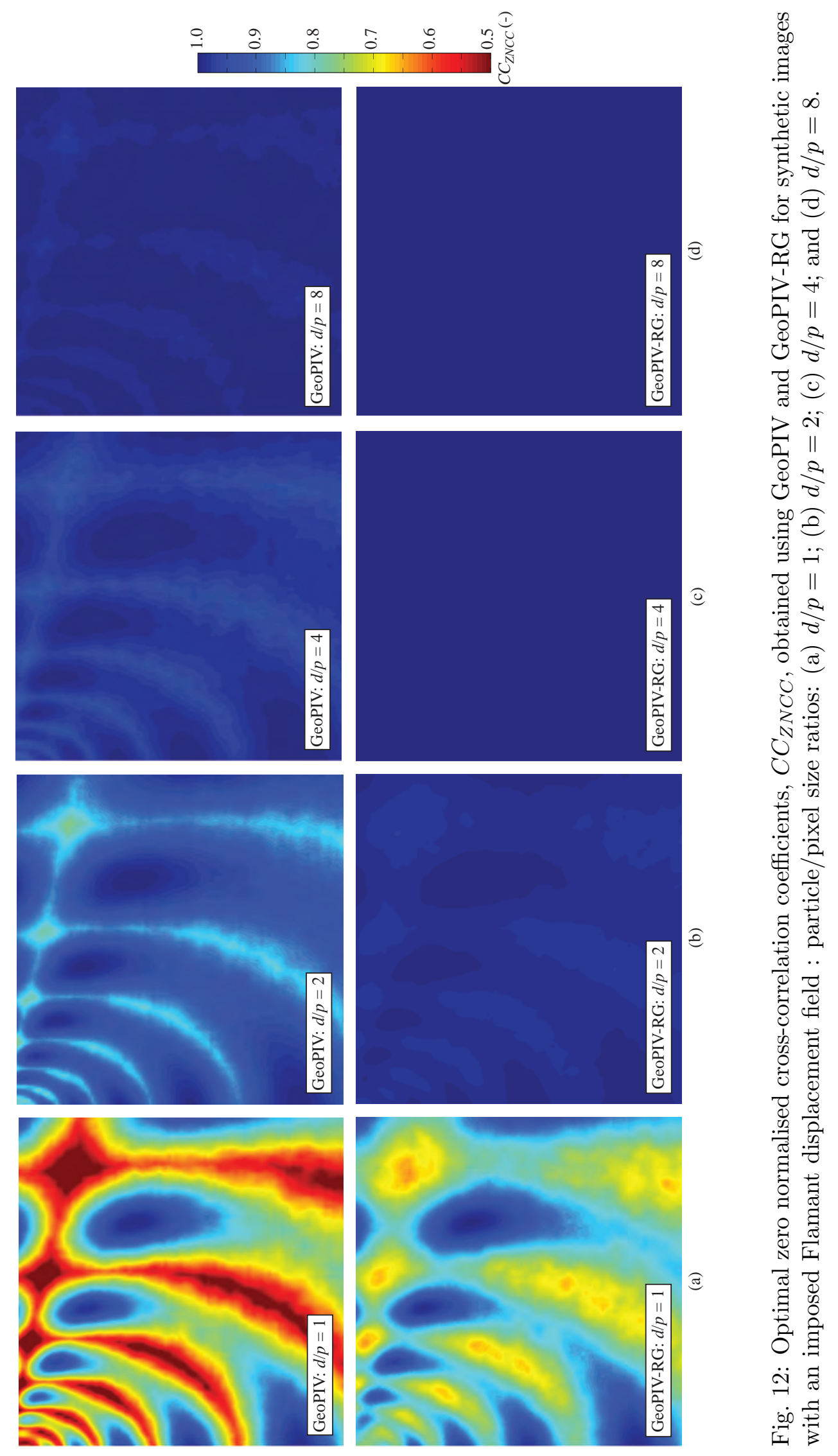




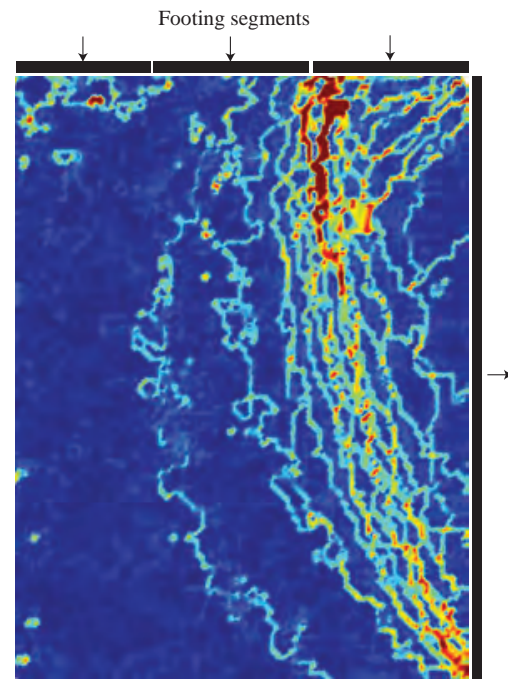

(a)

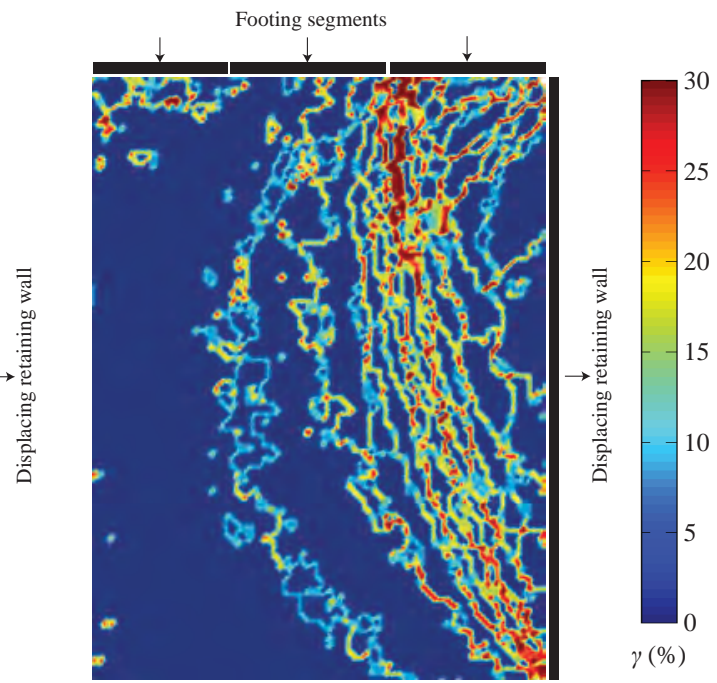

(b)

Fig. 13: Vermiculation identified in a model test of wall translation with surface footing load (Muir Wood \& Leśniewska, 2012): distribution of engineering shear strain shown (a) with and (b) without sub-pixel interpolation.

of the glass ballotini seen with transmitted light was used in PIV/DIC analysis of the displacement fields. In these images the contrast is lower than it might be if the pores were filled with air or water but the displacement fields deduced are much less influenced by possible wall friction because the received light has passed through the full thickness of the model ${ }^{1}$.

The original images analysed by Muir Wood \& Leśniewska (2012) were reanalysed for this paper using GeoPIV. Analyses, using $L_{s}=48 p$ and $s=12 p$, reproduce vermiculate patterns very similar to those shown by Muir Wood \& Leśniewska (2012), with minor differences resulting from the precise location of the PIV/DIC subsets distributed across the images (Figure 13).

Further analyses performed with different values of subset size, $L_{s}$, and spacing, $s$, indicated that the locations of the vermiculate features were insensitive to $L_{s}$ but that the strain level in these features was highly dependent upon the spacing, $s$, because strain is derived as a function of the gradient of displacement (rule R5) and the spacing $s$ provides the gauge length for strain calculation.

In this experiment the apparent particle to pixel ratio was $d / p \sim 10$, which would ordinarily indicate, according to Figure 7, that 'peak locking' would not occur. However, there are a number of other potential causes for the bias errors that cause the 'peak locking' effect seen in Figure 13 (a). One possibility relates

\footnotetext{
${ }^{1}$ As a relevant detailed comment, the later images were obtained using glass beads which had been annealed, leaving them essentially stress free, and making the model almost perfectly transparent. Previous tests had been performed using the same kind of glass beads, but in their original unannealed state. Thermal tempering (rapid cooling) of these particles left traces of tensile internal stress in each individual grain, making their assembly less transparent in ordinary light and much less transparent in polarised light.
} 


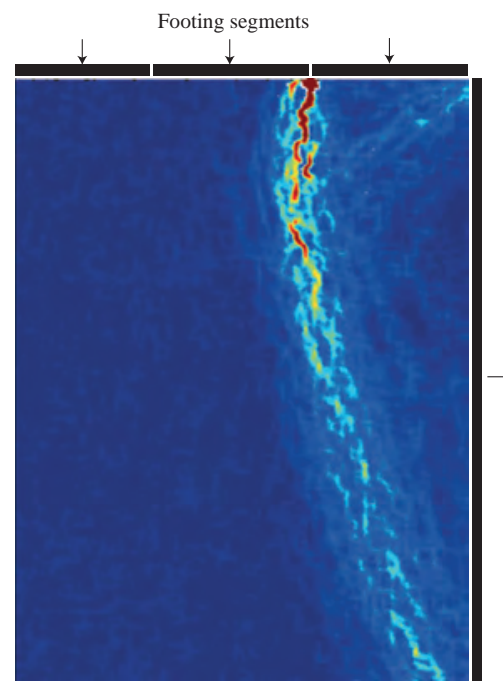

(a)

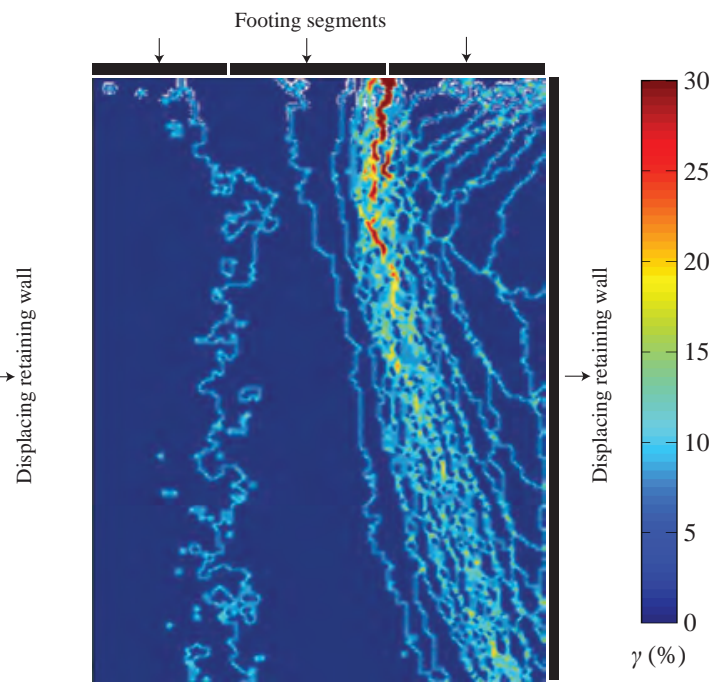

(b)

Fig. 14: Increment of wall displacement: distribution of engineering shear strain shown (a) with and (b) without sub-pixel interpolation. (Leśniewska \& Muir Wood, 2009):

to the quality of the image texture. To demonstrate this, images of a similar experiment, using the same apparatus and (unannealed) granular material but with a different digital camerapermitting better image texture ${ }^{2}$, were processed using the same analysis parameters. The engineering shear strain fields were again calculated from the displacement fields to sub-pixel and integer-pixel resolution and are shown in Figure 14. There are now fewer vermiculate features in the engineering shear strain fields where the displacements were refined to sub-pixel resolution (Figure 14 (a)) indicating that the sub-pixel refinement process is generally functioning quite well. The feature immediately beneath the left edge of the footing (which remains even with sub-pixel interpolation, Figure 14 (a)) may be spurious but this is a position in which localisation of strains is quite likely and the gradient of deformation is high. The improved image texture has suppressed the potential emergence of vermiculate features in the far-field.

The image texture in the subsets used to create Figure 13 was of low contrast. As a result the simple sub-pixel interpolation process has an insignificant effect on the calculated displacements which are all essentially integer pixel magnitudes: there is negligible difference between Figure 13 (a) and Figure 13 (b). This, combined with the large overlaps of subsets, leads to extreme strain localisation in these vermiculate features as a consequence of 'peak locking'.

How well the sub-pixel refinement process is working in these analyses can be quantified graphically by plotting histograms of the magnitudes of the displace-

\footnotetext{
${ }^{2} \mathrm{~A}$ different digital camera was also used in this experiment, however, it was of similar resolution to that used in the other experiment reported. Thus, the improvement in image texture evident in this experiment is thought to be due to the glass beads being left in their virgin, unannealed state.
} 


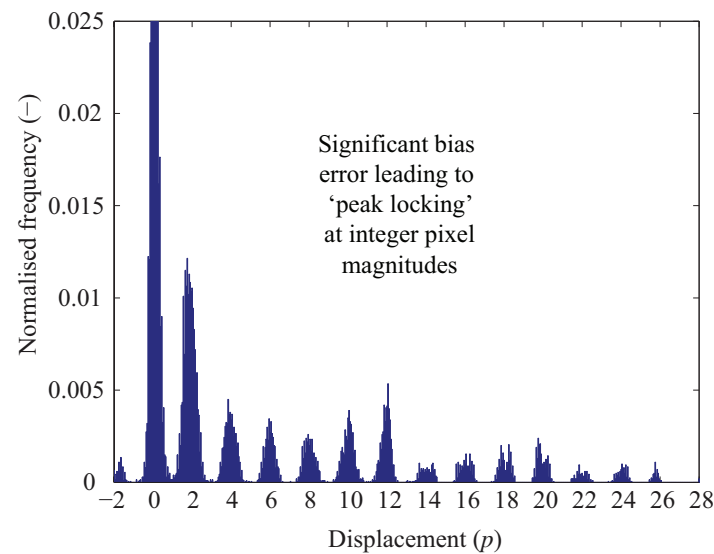

(a)

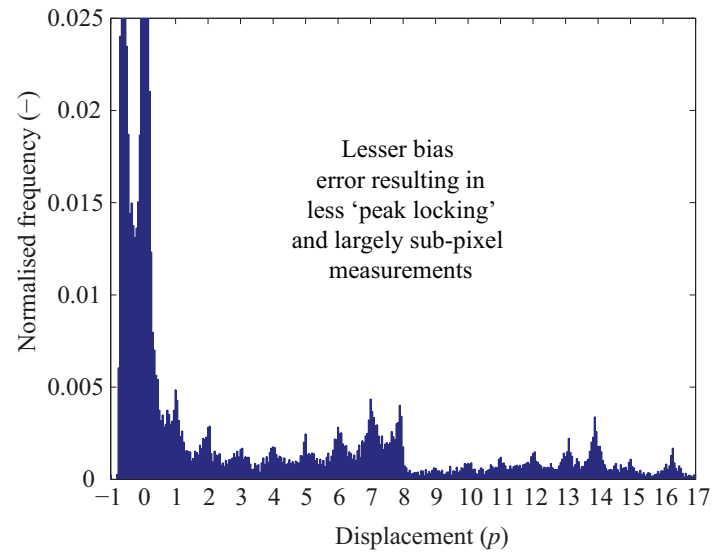

(b)

Fig. 15: Normalised histograms of displacement component magnitudes for model tests of wall/footing interaction: (a) clear vermiculation seen (Figure 13 (b)) (Muir Wood \& Leśniewska, 2012); and (b) no minimal vermiculation apparent (Figure 14 (a)) (Leśniewska \& Muir Wood, 2009).

ment components, as illustrated in Figure 15. Here, the frequencies have been normalised by $\sum n$ (where $n$ is the number of measurement points), because the different analyses used different numbers of subsets. It is clear that bias errors and 'peak locking' are occurring in both analyses, though to a much greater extent for the experiment with poor image texture (Figure 13, Figure 15(a)) than that with improved image texture (Figure 14, Figure 15 (b)).

The quality of the image texture has a strong effect on computation precision (Pan et al., 2008; Stanier \& White, 2013) and ought to be checked by calculating a measure of (i) global image quality via Mean Intensity Gradient (MIG) following Pan et al. (2010)Pan, Lu, \& Xie (2010), as set out by rule R1, and/or (ii) subset image quality by calculating the standard deviation of subset pixel intensities, $\sigma_{I_{s}}$ (Stanier \& White, 2013) or the Sum of Square of Subset Intensity Gradients (SSSIG) (Pan et al., 2008), as set out by rule R2. The values of $\sigma_{I_{s}}$ and $S S S I G$ for typical subsets confirm the importance of checking image texture quality (see Figure 16). The image texture in the experiment where vermiculation was observed (Figure 13) was sub-optimal (Figure 16(a)), contravening rules R1 and R2. In contrast, the image texture for the improved experiment, where the bias errors were less significant (Figure 14), was comparable to that for a geomaterial with optimised image texture (Stanier \& White, 2013) (compare Figure 16(b), (c)), so it was initially surprising that bias errors were still evident in the analysis of this image pair.

Persistent errors may also result if the magnitudes of displacement are large deformation are too large. eompared with the subset size (see Figure 15). This eontravenes rule R6 which proposes a limit on displacement of $\frac{1}{4} L_{s}$ precisely to avoid excessive subset deformation and rotation, which would in turn contravene rule R4 if the subset shape function were found to be of lower-order than the 


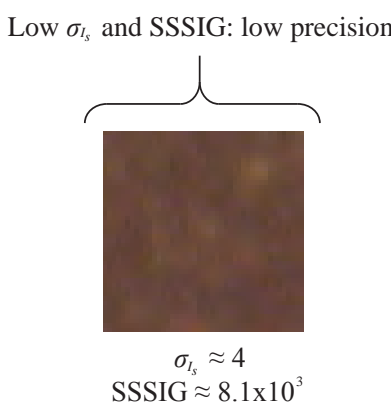

(a)

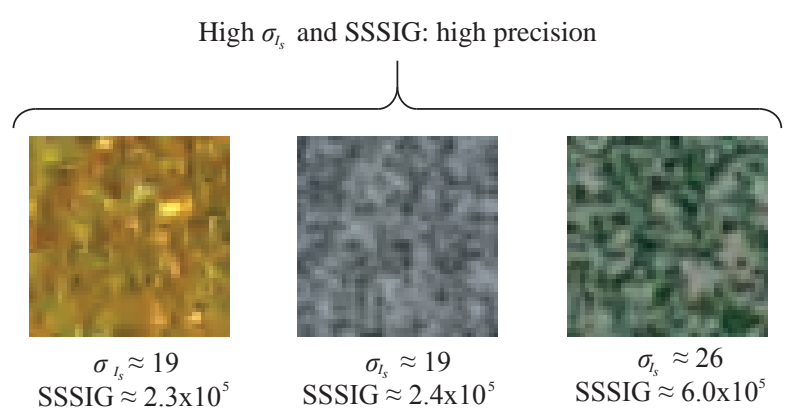

(b)

(c)

(d)

Fig. 16: Image texture for example subsets $(48 \times 48$ pixels $)$ and corresponding standard deviation of subset pixel intensities, $\sigma_{I_{s}}$, and $S S S I G$ for: (a) sub-optimal texture in a subset from the translating wall experiment reported by Muir Wood \& Leśniewska (2012); (b) excellent texture in a subset from a similar translating wall experiment described by Leśniewska \& Muir Wood (2009) with comparable quality to (c) optimally seeded sand and (d) optimally seeded clay as reported by Stanier \& White (2013).

subset deformation that is being measured. In both experiments the deformation imposed between the image pairs was relatively large (as indicated by engineering shear strains $\gamma>30 \%$ ) leading to probable contravention of $\mathrm{R} 6$ and potentially, if the subset shape function were found to be of lower-order than the subset deformation that is being measured, also rule R4. To check how these two effects were contributing to the errors the images from the two experiments were reinterpreted using GeoPIV-RG. The analysis settings adopted are summarised in Table 1.

The fields of engineering shear strain $\gamma$ presented in Figure $17(\mathrm{a}, \mathrm{b})$ show that the vermiculate features are largely suppressed (c.f. Figures 13 and 14 respectively), although there is more apparent noise than was seen in the analyses of the 'perfect' synthetic images. The optimised values of the cross-correlation coefficient $C C_{Z N C C}$ in Figure $17(\mathrm{c}, \mathrm{d})$ provide a check on the quality of the match between the locations of the subsets in the 'reference' and 'target' images after sub-pixel refinement. In the benchmarking of GeoPIV-RG, Stanier et al. (2015) suggest that a minimum value of $C C_{Z N C C}=0.75$ results in reliable displacement measurements that are practically unaffected by the loss of correlation caused by deformation. If the same criteria were applied to the analysis with clear vermiculations (Figure $17(\mathrm{a}, \mathrm{c})$ ) $86 \%$ of the measurement points would be discarded. In contrast, for the analysis with better image texture (Figure $17(\mathrm{~b}, \mathrm{~d})$ ) only $22 \%$ of the measurement points would be discarded by applying the same criterion, and most of these data points clearly lie in the region of intense straining swooping down from the edge of the footing. Evidently the better image texture leads to improved correlation and reduction in associated measurement errors.

Normalised histograms of the displacement components shown in Figure 18 (comparable to Figure 15) illustrate that for both experiments the bias errors are almost completely eradicated by the use of the first-order subset shape function of 


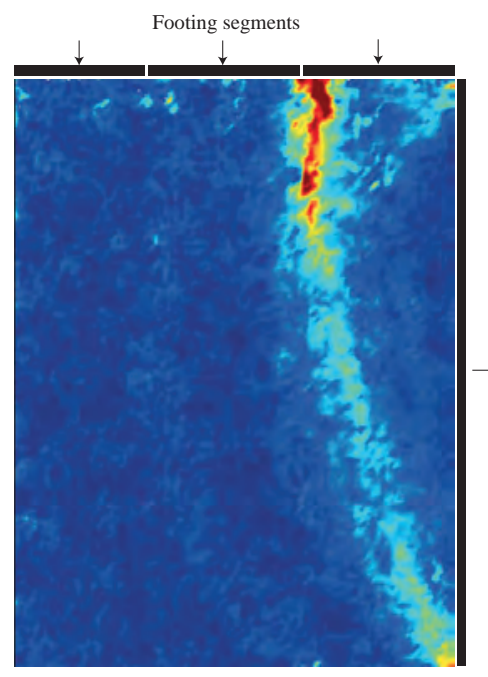

(a)

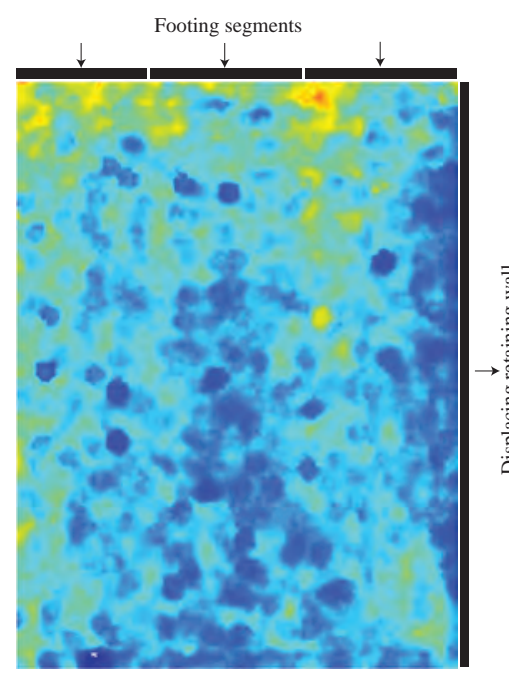

(c)

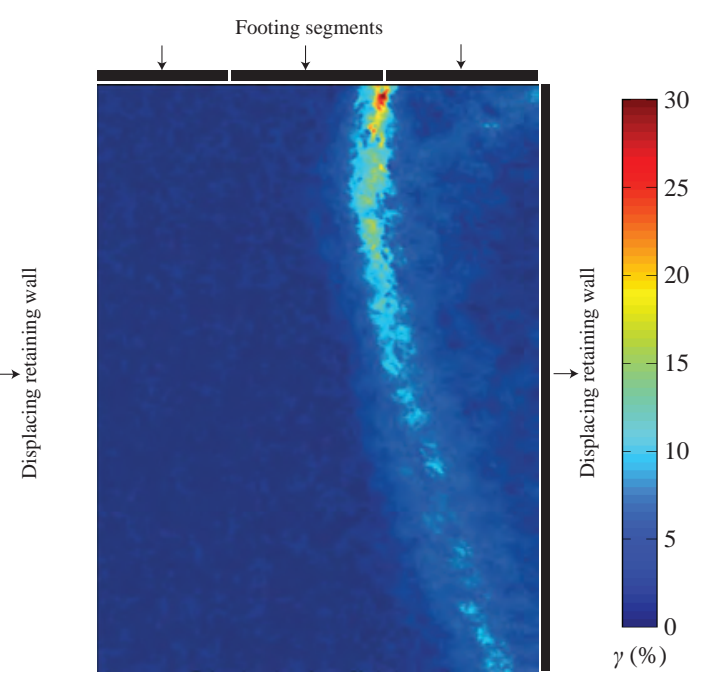

(b)

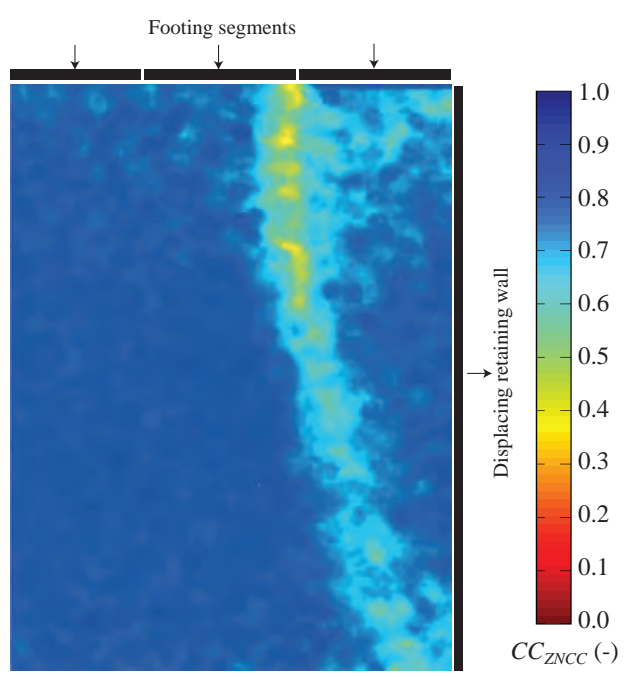

(d)

Fig. 17: Re-interpretation of the model tests of wall translation with surface footing load reported by (a, c) Muir Wood \& Leśniewska (2012) and (b, d) Leśniewska \& Muir Wood (2009) using GeoPIV-RG: (a,b) engineering shear strain, $\gamma$; and (c,d) optimal crosscorrelation coefficient, $C C_{Z N C C}$. 


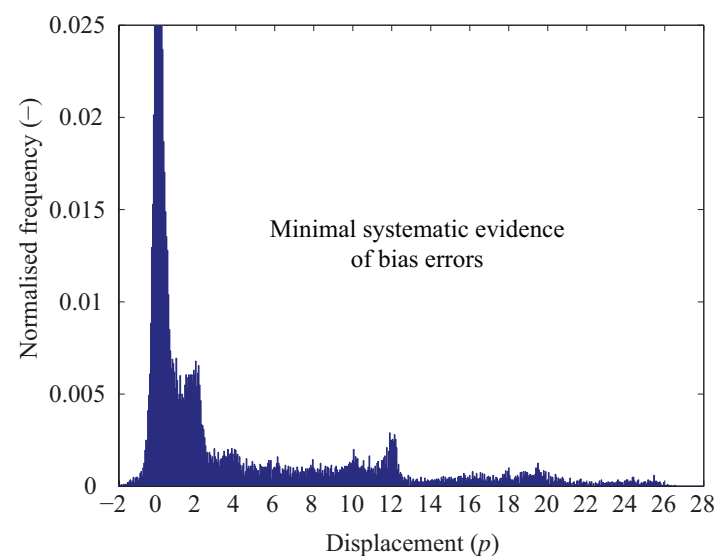

(a)

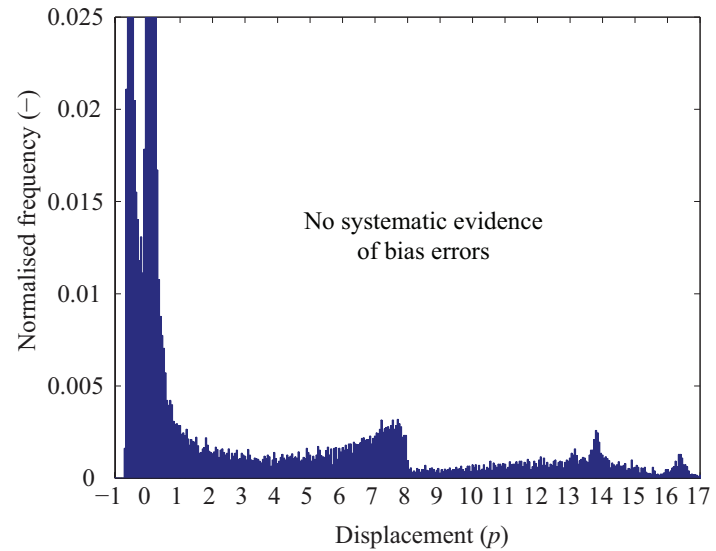

(b)

Fig. 18: Histograms of displacement component magnitudes for model tests of wall translation with surface footing load, re-analysed using GeoPIV-RG, for the model tests reported by: (a) Muir Wood \& Leśniewska (2012) and (b) Leśniewska \& Muir Wood (2009) (c.f. Figure 15 (a) and (b) respectively).

GeoPIV-RG. This supports the hypothesis that such higher order functions can be used for accurate determination of larger displacements deformations than can zero-order methods which use correlation peak interpolation for sub-pixel refinement.

To show (i) that these erroneous vermiculate features are amplified by increased overlapping when using GeoPIV, and (ii) that GeoPIV-RG is less susceptible to 'peak locking' for real data, a series of additional analyses with spacing $s=48 p$ (corresponding to overlapping ratio $\left.\left(\left(L_{s}-s\right) / L_{s}\right)=0\right)$ were performed. The resulting engineering shear strain fields are presented in Figure 19, side-by-side with those for $s=12 p$ (corresponding to overlap ratio of 0.75 ). For the model test reported by Muir Wood \& Leśniewska (2012), where the 'peak-locking' was extreme, the vermiculate features are still apparent for the zero-order GeoPIV analyses, even for $s=48 p$; only the magnitude of the features has changed (because the strain is calculated from the ratio of displacement step to subset spacing). In contrast, for the model test reported by Leśniewska \& Muir Wood (2009), where the 'peak-locking' was moderate, increasing the subset spacing (and thus reducing the overlap ratio) results in the masking of bias errors, as suggested by rule R5. For GeoPIV-RG the vermiculate features are suppressed for all cases, though it appears that detail is lost when the subset overlapping is reduced so that $s=48 p$. There are no major disadvantages in using quite large degrees of overlap in order to extract more detailed observations, so long as the underlying subset shape function is able to describe the deformations that have occurred. 


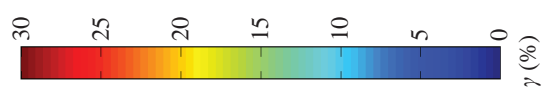

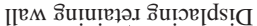

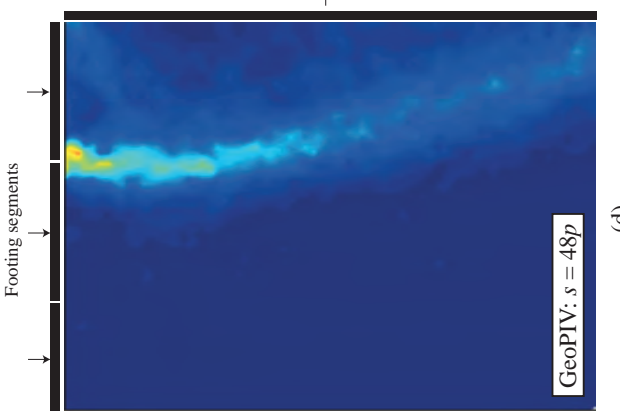

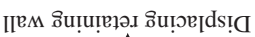

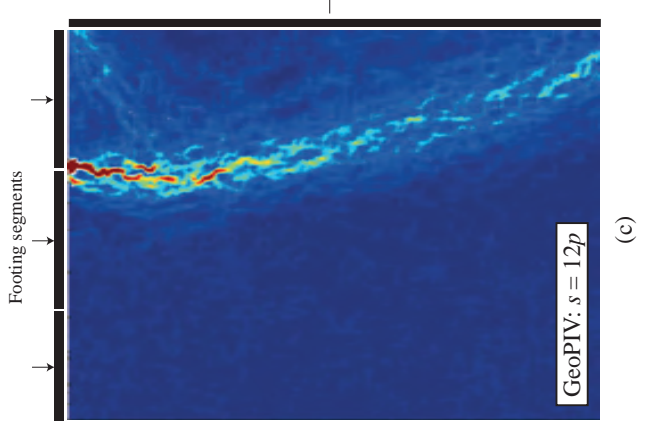

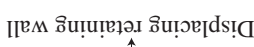

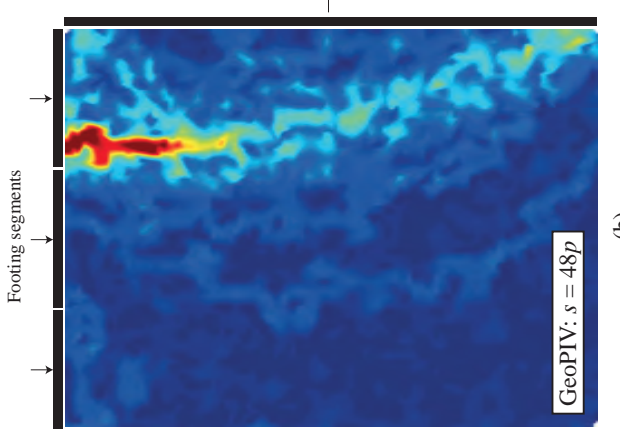

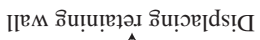

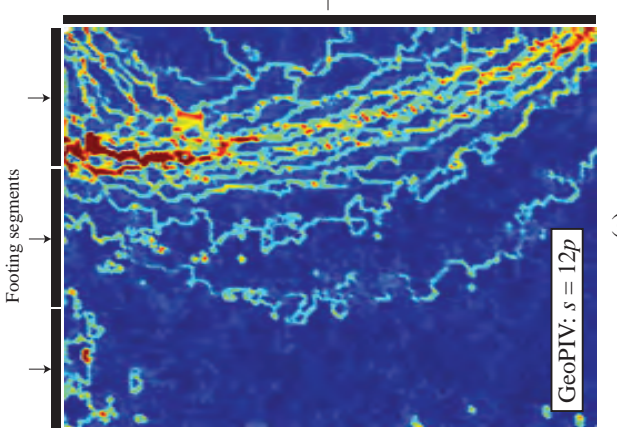

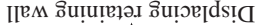

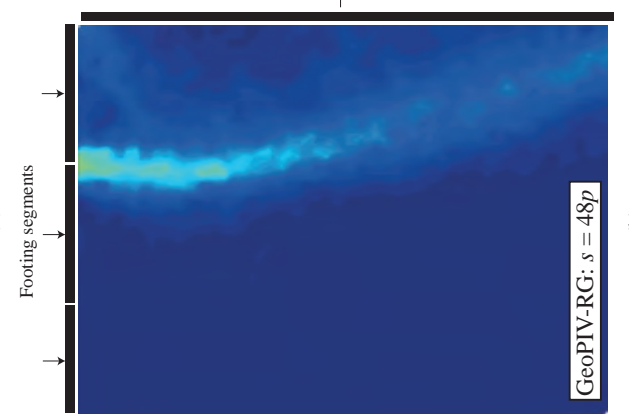

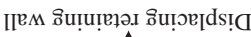

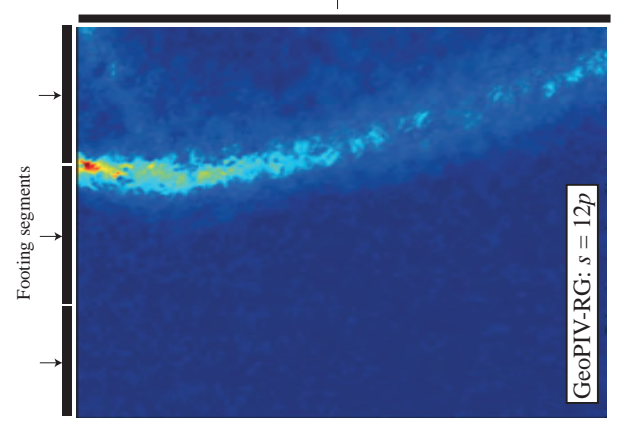

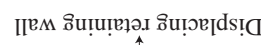

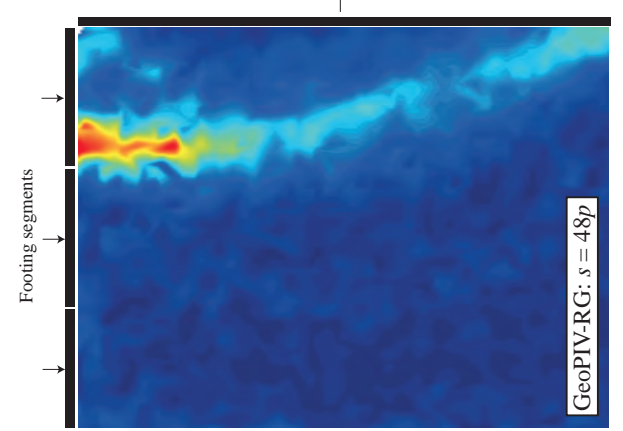

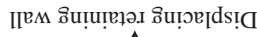

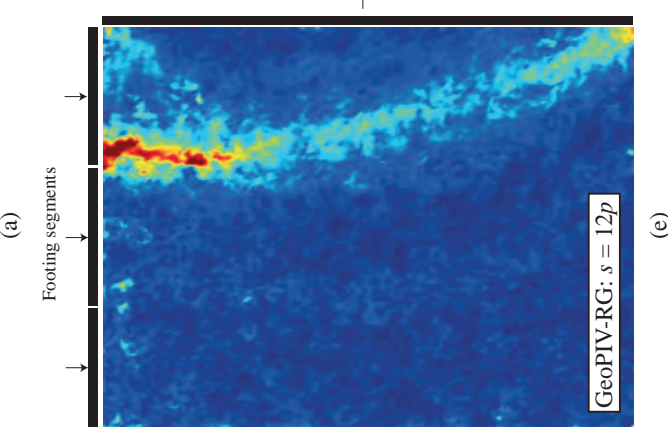

现.

की

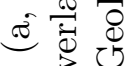

s.

ช

娄

पू $\infty 00$

응

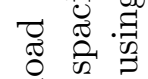

○)

.尹:

(ㅇํㅇ

$\stackrel{0}{8} 0$

ن

क ल

站

उक्ष

च के

로용

$\overbrace{i=1}=$

$=$

पे

os

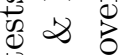

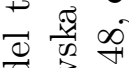

.

घี ब्नु क

임.

工ิ

क्षे की

$\therefore+\overrightarrow{0}$

हैं

可

$\exists$

สี ช

पू

$\because \sqrt{*}$

焉

$3 \ldots$

舟

च्चु

\&્⿹

क्ष०त 20

$=\infty$ के

되뭉츠

$\ddot{2}$ की

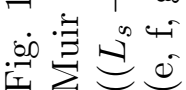




\section{Conclusions}

This paper has explored the possibility that vermiculate features observed in image analyses of some experiments involving deformation of granular materials might be spurious artefacts. Analyses of synthetic images, and reinterpretation of image pairs from experiments using state-of-the-art image analysis algorithms, have demonstrated that the vermiculate features are almost certainly the product of a combination of both the experimental setup and shortcomings in the image analysis algorithms originally employed.

The studies of synthetic and real images of granular materials have demonstrated that an improvement of performance can be obtained using computationally more intensive algorithms which apply higher order shape functions to describe the deformations occurring within the analysis subsets. However, while such algorithms can suppress the appearance of spurious vermiculate features, the quality of the resulting measurements is still compromised if the image texture is of suboptimal quality. If the particles are too small or are not easily distinguished from the background, then their movement cannot be reliably detected no matter how sophisticated the subsequent image analysis. The resulting bias errors become more dramatic when strains are calculated from derivatives of the displacements. Subset overlapping can also lead to the amplification of resulting errors and, in some cases, to the generation of spurious strain features.

There are several specific recommendations:

1. Choose seeding particles that are of sufficient diameter within the images captured for each experimental setup (i.e. d $/ p>4$ ).

2. Seed the exposed plane of geotechnical models with dyed sand or modelling flock such that $0.3<A S R<0.7$, so as to ensure that an optimal amount of seeding is applied to each model.

3. Evaluate the quality of the images to be analysed using appropriate statistical measures of eontrast and signal/noise ratioimage texture quality (e.g. $M I G, S S S I G$ and $\left.\sigma_{I_{s}}\right)$.

4. Inspect the histogram of displacements to ensure no integer pixel bias.

5. Make use of image analysis software that incorporates higher-order subset deformation functions.

6. Explore the effect of varying the spacing of correlation subsets.

7. Confirm the mechanical plausibility of the patterns of displacement and strain that emerge from the image analysis.

Users of advanced image correlation techniques need to be aware that spurious numerical artefacts can be unwittingly generated in the computed strain fields. Such awareness should then prompt them to consider the potential for introduction of improvements at all stages of the image acquisition and processing chain. 
Guidance has been given concerning details of the experimental equipment and procedures and concerning the selection and application of the image analysis algorithms. As is often the case, apparently simple-to-use but sophisticated programs contain hidden traps for the unwary.

\section{Acknowledgements}

The work forms part of the activities of the Centre for Offshore Foundation Systems (COFS) at the University of Western Australia, which is supported by the Lloyd's Register Foundation as a Centre of Excellence and is a node of the Australian Research Council (ARC) Centre of Excellence in Geotechnical Science and Engineering. Jim Hambleton was seconded to University of Western Australia under the aegis of the ARC Centre of Excellence, Geotechnical Science and Engineering. Jelke Dijkstra is supported by an Intra-European Fellowship EC/FP7MC-IEF 623613 EMPIRC. Danuta Leśniewska was supported by NCN grant no 2011/03/B/ST8/05865. David White holds the Shell Energy and Minerals Institute (EMI) Chair in Offshore Engineering. David Muir Wood is particularly grateful for the support of the Centre for Offshore Foundation Systems, University of Western Australia through the award of the Martin Fahey Visiting Fellowship during 2013. He also acknowledges the support of the Northern Research Partnership of Scottish universities at Dundee University and the invaluable support of the British Council in making initial experimental contacts for photoelastic and image analysis of granular materials.

\section{References}

Adrian, R. \& Westerweel, J. (2011). Particle image velocimetry. Cambridge University Press.

Anastasopoulos, I., Gazetas, M., Bransby, M., Davies, M., \& El Nahas, A. (2007). Fault rupture propagation through sand: finite-element analysis and validation through centrifuge experiments. Journal of Geotechnical and GeoEenvironmental Engineeering 133, 943-958.

Ando, E., Hall, S., Viggiani, G., Desrues, J., \& Bésuelle, P. (2012). Grain-scale experimental investigation of localised deformation in sand: a discrete particle tracking approach. Acta Geotechnica 7(1), 1-13.

Bhandari, A., Powrie, W., \& Harkness, R. (2012). A digital image-based deformation measurement system for triaxial tests. ASTM Geotechnical Testing Journal 35(2), 209-226.

Blaber, J., Adair, B., \& Antoniou, A. (2015). Ncorr: open-source 2d digital image correlation matlab software. Journal of Experimental Mechanics, published ahead of print online. 
Cosman, P., Gray, R., \& Olshen, R. (1994). Evaluating quality of compressed medical images: SNR, subjective rating, and diagnostic accuracy. Proceedings of the IEEE 82(6), 919-932.

Crocker, J. \& Grier, D. (1996). Methods of digital video microscopy for colloidal studies. Journal of Colloid and Interface Science 179(1), 298-310.

Flamant, A. (1892). Sur la réparation des pressions dans un solide rectangulaire chargé transversalement. Comptes Rendus de l'Académie des Sciences 114, 1465-1468.

Gui, L. \& Wereley, S. (2002). A correlation-based continuous window-shift technique to reduce the peak-locking effect in digital PIV image evaluation. Experiments in Fluids 32(4), 506-517.

Hall, S., Bornert, M., Desrues, J., Pannier, Y., Lenoir, N., Viggiani, G., \& Bésuelle, P. (2010). Discrete and continuum analysis of localised deformation in sand using X-ray $\mu$ ct and volumetric digital image correlation. Géotechnique 60(5), 315-322.

Keshavarzy, A. \& Ball, J. (1999). An application of image processing in the study of sediment motion. Journal of Hydraulic Research 37(4), 559-576.

Lee, C., Take, W., \& Hoult, N. (2012). Optimum accuracy of two-dimensional strain measurements using digital image correlation. Journal of Computing in Civil Engineering 26, 795-803.

Leśniewska, D. \& Muir Wood, D. (2009). Observations of stresses and strains in a granular material. Journal of Engineering Mechanics 135(9), 1038-1054.

Lewis, J. (1995). Fast normalized cross-correlation. Vision Interface 10(1), 120 123.

Marshall, A., Farrel, R., Klar, A., \& Mair, R. (2012). Tunnels in sands: the effect of size, depth and volume loss on greenfield displacements. Géotechnique 62(5), 385-399.

Muir Wood, D. \& Leśniewska, D. (2012). Discussion: Heterogeneity and soil element testing. Géotechnique Letters 2(5), 217-219.

Nazhat, Y. \& Airey, D. (2015). The kinematics of granular soils subjected to rapid impact loading. Granular Matter 17(1), 1-20.

Pan, B., Li, K., \& Tong, W. (2013). Fast, robust and accurate digital image correlation calculation without redundant computations. Experimental Mechanics 53(7), 1277-1289. 
Pan, B., Lu, Z., \& Xie, H. (2010). Mean intensity gradient: an effective global parameter for quality assessment of the speckle patterns used in digital image correlation. Optics and Lasers in Engineering 48(4), 469-477.

Pan, B., Wu, D., \& Xia, Y. (2012). Incremental calculation for large deforamtion measurement. Optics and Lasers in Engineering 50, 586-592.

Pan, B., Xie, H., \& Wang, Z. (2010). Equivalence of digital image correlation criteria for pattern matching. Applied Optics 49(28), 5501-5509.

Pan, B., Xie, H., Wang, Z., Qian, K., \& Wang, Z. (2008). Study on subset size selection in digital image correlation for speckle patterns. Optics Express 16(10), 7037-7048.

Raffel, M., Willert, C., Wereley, S., \& Kompenhans, J. (2007). Particle Image Velocimetry: a practical guide. Springer.

Rechenmacher, A. \& Finno, R. (2004). Digital image correlation to evaluate shear banding in dilative sands. Geotechnical Testing Journal 27(1), 1-10.

Rosenbrand, E. \& Dijkstra, J. (2012). Application of image subtraction data to quantify suffusion. Géotechnique Letters 2, 37-41.

Scarano, F. (2013). Tomographic PIV: principles and practice. Measurement Science and Technology 24(1), 012001.

Schreier, H. \& Sutton, M. (2002). Systematic errors in digital image correlation due to under matched subset shape functions. Journal of Experimental Mechanics 42(3), 303-310.

Stanier, S., Blaber, J., Take, W., \& White, D. (2015). Improved image-based deformation measurement for geotechnical applications. Canadian Geotechnical Journal, accepted.

Stanier, S. \& White, D. (2013). Improved image-based deformation measurement in the centrifuge environment. ASTM Geotechnical Testing Journal 36(6), 915928.

Stelzer, E. (1998). Contrast, resolution, pixelation, dynamic range and signal-tonoise ratio: fundamental limits to resolution in fluorescence light microscopy. Journal of Microscopy 189(1), 15-24.

Sutton, M. (2008). Digital image correlation for shape and deformation measurements. In W. Sharpe Jr. (Ed.), Springer Handbook of Experimental Solid Mechanics, Chapter 20. Springer.

Sutton, M., Wolters, W., Peters, W., Ranson, W., \& McNeill, S. (1983). Determination of displacements using an improved digital correlation method. Image and Vision Computing 1(3), 133-139. 
Sveen, J. (2004). An introduction to MATPIV v. 1.6. 1. Eprint no. 2, issn 08094403, Dept. of Mathematics, University of oslo.

Take, W. \& Bolton, M. (2004). Identification of seasonal slope behaviour mechanisms from centrifuge case studies. Proceedings of the Conference on Advances in Geotechnical Engineering: The Skempton Conference, 992-1004.

Taylor, Z., Gurka, R.vand Kopp, G., \& Liberzon, A. (2010). Long-duration time-resolved PIV to study unsteady aerodynamics. IEEE Transactions on Instrumentation and Measurement 59(12), 3262-3269.

Thielicke, W. \& Stamhuis, E. (2014). PIVlab-towards user-friendly, affordable and accurate digital Particle Image Velocimetry in MATLAB. Journal of Open Research Software 2(1), e30.

Tong, W. (2005). An evaluation of digital image correlation criteria for strain mapping applications. Strain 41(4), 167-175.

Vennemann, P. (2015). JPIV online manual: http://www.jpiv.vennemannonline.de.

Westerweel, J. (1997). Fundamentals of digital particle image velocimetry. Measurement Science and Technology 8(12), 1379.

Westerweel, J. (2000). Effect of sensor geometry on the performance of PIV interrogation. In Laser techniques applied to fluid mechanics, pp. 37-55. Springer.

White, D. \& Bolton, M. (2004). Displacement and strain paths during planestrain model pile installation in sand. Géotechnique 54(6), 375-397.

White, D., Take, W., \& Bolton, M. (2003). Soil deformation measurement using particle image velocimetry (PIV) and photogrammetry. Géotechnique 53(7), 619-631.

Yu, L. \& Pan, B. (2015). The errors in digital image correlation due to overmatched shape functions. Measurement Science and Technology 045202, 1-9.

\section{Appendix: definitions of image analysis variables}

For completeness the various variables that have been mentioned are briefly defined here.

The zero normalised cross-correlation coefficient $\left(C C_{Z N C C}\right)$ is defined as:

$$
C C_{Z N C C}=\frac{\sum_{i, j \in n_{s}}\left(I_{i j}^{r}-\bar{I}^{r}\right)\left(I_{i, j}^{t}-\bar{I}^{t}\right)}{\sqrt{\sum_{i, j \in n_{s}}\left[I_{i, j}^{r}-\bar{I}^{r}\right]^{2} \sum_{i, j \in s}\left[I_{i, j}^{t}-\bar{I}^{t}\right]^{2}}}
$$


where $I_{i, j}^{r}$ and $I_{i, j}^{t}$ are the pixel intensities for a given $i, j$ coordinate in the 'reference' and 'target' images respectively. The mean pixel intensities for the 'reference' and 'target' images, $\bar{I}^{r}$ and $\bar{I}^{t}$ are calculated as

$$
\bar{I}^{r}=\frac{1}{n_{s}} \sum_{i, j \in n_{s}} I_{i, j}^{r} ; \quad \bar{I}^{t}=\frac{1}{n_{s}} \sum_{i, j \in n_{s}} I_{i, j}^{t}
$$

where $n_{s}$ is the number of pixels in the subset.

The Signal to Noise Ratio $S N R$ is defined as:

$$
S N R=\frac{I_{\text {signal }}}{I_{\text {noise }}}
$$

where $I_{\text {signal }}$ and $I_{\text {noise }}$ are the intensity components representing the signal and the noise respectively.

The Mean Intensity Gradient MIG is defined (after Pan, Lu, \& Xie (2010)) as:

$$
M I G=\sum_{i, j \in n_{I}} \frac{1}{n_{I}} \sqrt{\left(I_{x}^{\prime}\right)^{2}+\left(I_{y}^{\prime}\right)^{2}}
$$

where $I_{x}^{\prime}$ and $I_{y}^{\prime}$ are the intensity gradients in the $x$ and $y$ directions respectively and $n_{I}$ is the number of pixels in the image.

The Artificial Seeding Ratio $A S R$ is defined (after Stanier \& White (2013)) as:

$$
A S R=\frac{\bar{I}-\bar{I}_{A S R=0}}{\bar{I}_{A S R=1}-\bar{I}_{A S R=0}}
$$

where $\bar{I}$ is the mean pixel intensity of the image, $\bar{I}_{A S R=0}$ is the mean pixel intensity for an image with no artificial seeding and $\bar{I}_{A S R=1}$ is the mean pixel intensity for an image with saturated artificial seeding.

The standard deviation of the subset pixel intensities $\sigma_{I_{s}}$ is computed as:

$$
\sigma_{I_{s}}=\sqrt{\frac{1}{n_{s}} \sum_{i, j \in n_{s}}\left(I_{i, j}-\bar{I}\right)^{2}}
$$

where $I_{i, j}$ and $\bar{I}$ are the pixel and mean subset pixel intensities respectively and $n_{s}$ is the number of pixels in the subset.

The Sum of Squares of Subset pixel Intensity Gradients (SSSIG) are defined (after Pan et al. (2008)) as:

$$
\begin{aligned}
& S S S I G_{x}=\sum_{i, j \in n_{s}}\left(I_{x}^{\prime}\right)^{2} \\
& S S S I G_{y}=\sum_{i, j \in n_{s}}\left(I_{y}^{\prime}\right)^{2}
\end{aligned}
$$


where $I_{x}^{\prime}$ and $I_{y}^{\prime}$ are as defined previously and $n_{s}$ is the number of pixels in the subset.

The intensity of each pixel in the artificial images was generated using the following function:

$$
I_{i, j}=\min \left(\sum_{n=1}^{n_{p}} C \exp \left(\frac{-\left(i-x_{p}(n)\right)^{2}-\left(j-y_{p}(n)\right)^{2}}{\left(d_{p} / 2\right)^{2}}\right), 255\right)
$$

where $I_{i, 2}$ is the intensity of the pixel in row $i$ and column $j$ of the artifical image, $n_{2}$ is the total number of projected dots and $n$ is the $n^{\text {th }}$ projected dot. $C$ is the contrast of the dots in the range of 0-255 (in this instance $C$ was taken as 200), $x_{p}(n)$ and $y_{p}(n)$ are the coordinates of the $n^{\text {th }}$ dot (taken as the central pixel for the single dot in the images in Figure 6 and randomly generated for each of the dots in the images in Figure 9) and $d_{2}$ is the diameter of the projected dots. The intensity of each pixel $I_{i, j}$ was capped at 255 since that is the maximum brightness of a pixel in an 8-bit digital image. 
LaTeX Source Files
Click here to download LaTeX Source Files: LaTeX Source Files.zip

LaTeX Source Files
Click here to download LaTeX Source Files: LaTeX Source Files.zip Click here to download LaTeX Source Files: LaTeX Source Files.zip

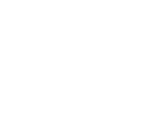
(1) . .

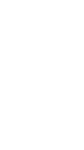

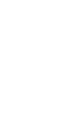
(1) (1) (n) (1)

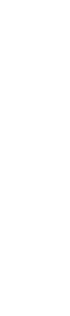

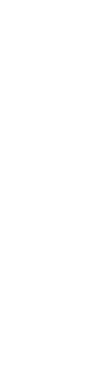

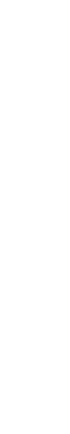
更

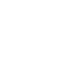
更

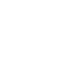
更 更 (1) 